\title{
Annotated Checklist of the Fishes of Wake Atoll ${ }^{1}$
}

\author{
Pbillip S. Lobel ${ }^{2}$ and Lisa Kerr Lobel ${ }^{3}$
}

\begin{abstract}
This study documents a total of 321 fishes in 64 families occurring at Wake Atoll, a coral atoll located at $19^{\circ} 17^{\prime} \mathrm{N}, 166^{\circ} 36^{\prime} \mathrm{E}$. Ten fishes are listed by genus only and one by family; some of these represent undescribed species. The first published account of the fishes of Wake by Fowler and Ball in 1925 listed 107 species in 31 families. This paper updates 54 synonyms and corrects 20 misidentifications listed in the earlier account. The most recent published account by Myers in 1999 listed 122 fishes in 33 families. Our field surveys add 143 additional species records and 22 new family records for the atoll. Zoogeographic analysis indicates that the greatest species overlap of Wake Atoll fishes occurs with the Mariana Islands. Several fish species common at Wake Atoll are on the IUCN Red List or are otherwise of concern for conservation. Fish populations at Wake Atoll are protected by virtue of it being a U.S. military base and off limits to commercial fishing.
\end{abstract}

Wake Atoll is an isolated atoll in the central Pacific $\left(19^{\circ} 17^{\prime} \mathrm{N}, 166^{\circ} 36^{\prime} \mathrm{E}\right)$. It is approximately $3 \mathrm{~km}$ wide by $6.5 \mathrm{~km}$ long and consists of three islands with a land area of approximately $6.5 \mathrm{~km}^{2}$. Wake is separated by $546 \mathrm{~km}$ of deep ocean from the nearest reef system on Taongi Atoll in the Marshall Islands to the south. It is $1900 \mathrm{~km}$ southwest of Midway Atoll, $1400 \mathrm{~km}$ southeast of Minami-tori-shima (Marcus) Island, and 2260 $\mathrm{km}$ east of the Mariana Islands. Thus, it is in a unique position with respect to the biogeography of reef fauna. This survey fills a major data gap in the zoogeography of Pacific fishes (see Randall 1998).

Wake Atoll is a U.S. Air Force installation administered by the U.S. Army Space

${ }^{1}$ This project was supported by the Department of Defense Legacy Resource Management Program grants DACA87-97-H-0006 and DACA87-01-H-00013 and by the Army Research Office grants DAAG55-98-1-0304 and DAAD19-02-1-0218. Manuscript accepted 9 May 2003.

2 Boston University Marine Program, Marine Biological Laboratory, Woods Hole, Massachusetts 02543.

${ }^{3}$ Department of Biology, University of Massachusetts, 100 Morrissey Boulevard, Boston, Massachusetts 02125 .

Pacific Science (2004), vol. 58, no. 1:65-90

(C) 2004 by University of Hawai'i Press

All rights reserved and Strategic Defense Command. Consequentially, access has been limited due to the military mission, and as a result the aquatic fauna of the atoll has not received thorough investigation. Our surveys were sponsored by the Department of Defense Legacy Resource Management Program as part of the program to implement coral reef conservation and protection (Kerr and Lobel 2000).

The first ichthyological collection at Wake resulted in a checklist of 107 species (Fowler and Ball 1925). Specimens were deposited in the Bernice P. Bishop Museum (врвм), Honolulu, Hawai $i$, with duplicates deposited in the Academy of Natural Sciences in Philadelphia (ANSP). The second important collection was by W. Gosline and J. Randall during 1953; most of their specimens are also deposited in the врвм with a few at the National Museum of Natural History (USNM) and the remaining specimens are held in the University of Hawai'i (Department of Zoology) fish collection. Specimens held in the California Academy of Sciences (CAS) were collected by J. Kauanui and P. Kauanui or J. Oetzel in 1956 and 1957. Specimens held in the Harvard Museum of Comparative Zoology (MCZ) were collected by us in 1999. The collections of the Tanager Expedition and by Gosline and Randall were primarily in shallow water without the benefit of scuba and based largely on the use of ichthyocides and conventional 
fishing techniques. Myers' (1999) listing of fishes from Wake Atoll is based on photographs, or in some cases video, taken by military personnel.

This checklist includes all species reported in previous publications, species not previously verified or published from the $\mathrm{B}$. $\mathrm{P}$. Bishop Museum collection, and new records found during our comprehensive scuba surveys conducted at Wake during July 1997 and May 1999. We have updated species reported in previous publications with current nomenclature and list the synonyms.

The following checklist represents all of the fishes currently known to occur at Wake Atoll. A total of 143 new records is added and includes fishes from the following families: Rhincodontidae, Albulidae, Moringuidae, Chlopsidae, Muraenidae (eight species), Ophichthidae (two species), Congridae (two species), Bythitidae, Mugilidae (three species), Atherinidae, Exocoetidae, Holocentridae (three species), Fistularidae, Scorpaenidae (five species), Caracanthidae, Serranidae (eight species), Cirrhitidae, Pseudochromidae, Priacanthidae, Apogonidae (eight species), Malacanthidae, Echeneidae, Carangidae (six species), Lutjanidae (two species), Lethrinidae, Mullidae (three species), Pempheridae, Chaetodontidae (two species), Pomacanthidae, Oplegnathidae, Pomacentridae (six species), Labridae (18 species), Scaridae (three species), Pinguipedidae, Creedidae, Tripterygidae, Blenniidae (three species), Callionymidae, Gobiidae (14 species), Microdesmidae, Siganidae, Acanthuridae (eight species), Bothidae, Balistidae (three species), Monacanthidae (two species), and Tetraodontidae (four species). Twenty-two of these families represent new family records for Wake Atoll.

\section{MATERIALS AND METHODS}

Species are listed in phylogenetic sequence by family with the current valid name first, followed by synonyms if they were cited in other checklists. An asterisk $\left(^{*}\right)$ designates those species that are our new records for Wake Atoll. The author and date of description are given for each species followed by the record(s) for Wake Atoll. Museum specimen numbers (i.e., врвм 1234) are given for those species maintained in ichthyological collections. In addition, the expedition during which the specimens in the Bishop Museum were collected is designated: by (T) for the Tanager Expedition of 1923, (K) for fishes collected by J. Kauanui in 1957, (G\&R) for fishes collected by Gosline, Randall et al. during 1953, and (K\&L) for fishes collected by us in 1997 and 1999 .

Lists of specimens from other scientific collections at Wake Atoll but that have not yet been published were provided by the ANSP, вРвм, and CAs. The list of fishes from the USNM was taken from their Web site (http://www.nmnh.si.edu/vert/fishcat/).

The identification of many of the specimens held in the врвм was verified by $\mathrm{J}$. Randall and A. Suzumoto. We verified the identity of the specimens held in the University of Hawai'i, Department of Zoology fish collection. J. McCosker verified some of the eel specimens held in the cas; however, many of the specimens reported in the collections of CAS, USNM, and ANSP were not reexamined. Thus upon further taxonomic evaluation, some of these earlier identifications may be revised.

Our underwater surveys were conducted during two expeditions: 10 to 23 July 1997 and 15 to 29 May 1999. We completed a total of 118 dives during that time at sites located around the atoll (Figure 1). In addition, more than a dozen shallow-water snorkel surveys were also conducted at locations around the atoll. The majority of observations were done by visual and photographic census (using both still and video photography, which are stored in our collection). Several collections of small cryptic fishes were made using quinaldine. These were all located at the dive site directly off the small boat harbor and ranged in depth from 10 to $50 \mathrm{~m}$. Several fishes were speared or collected by nets to confirm species identification.

Overall, our surveys provide a reliable census for the majority of reef fishes but certainly did not adequately sample small cryptic species that remain hidden in crevices and corals. Future surveys should include sampling the cryptic fauna. 


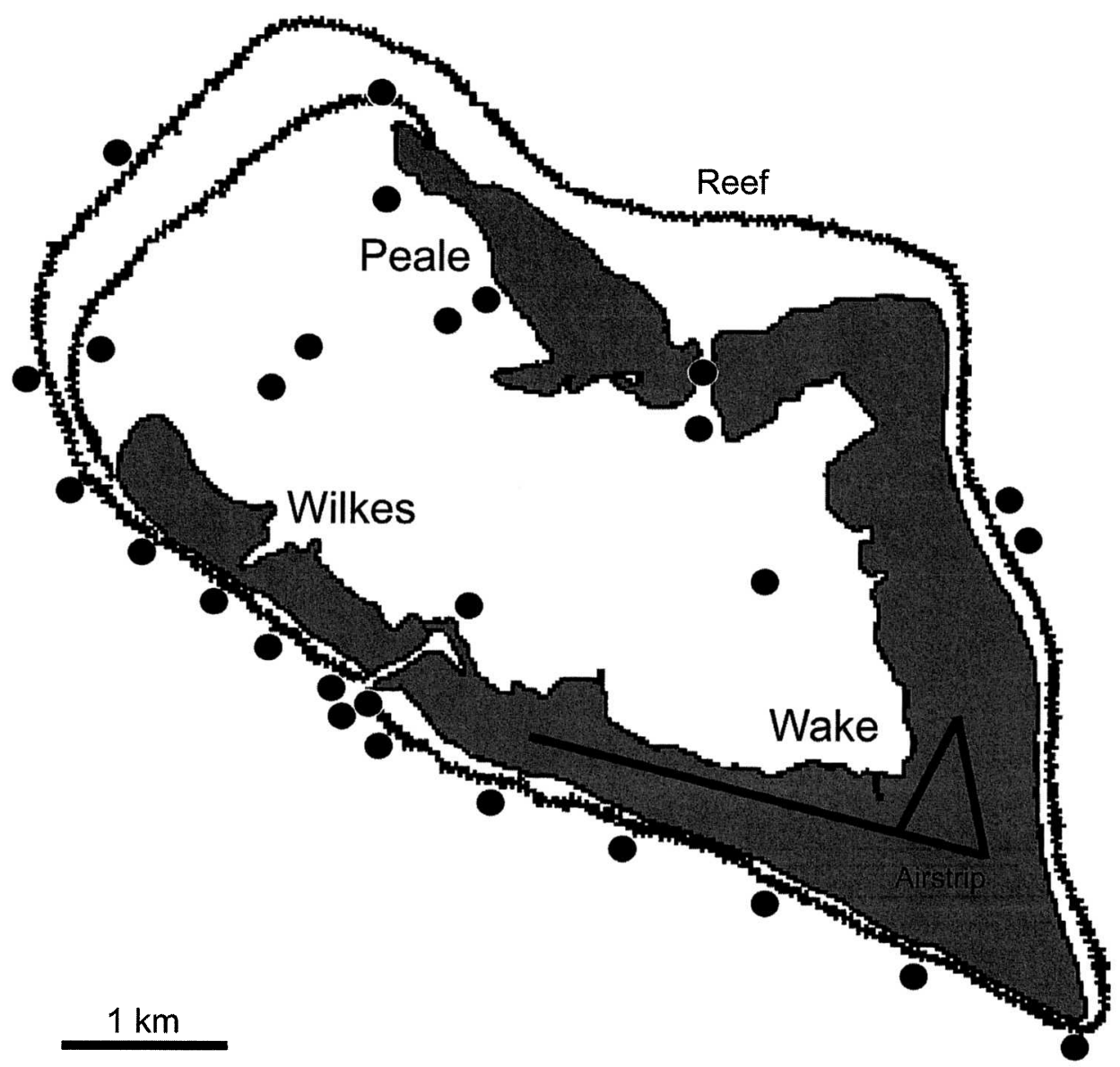

FIGURE 1. Fish survey locations around Wake Atoll. Black dots show survey sites; multiple dives were conducted at many of these locations.

RESULTS

Family Rhincodontidae (Whale Shark)

${ }^{*}$ Rbincodon typus (Smith, 1828). Sight record reported by island divers.

Family Carcharhinidae (Requiem Sharks)

Carcharbinus amblyrbynchos (Bleeker, 1856). Myers 1999.

Carcharbinus melanopterus (Quoy \& Gaimard, 1824). Myers 1999. No blacktip reef sharks were observed by us.

Eulamia commersoni (Blainville). врвм 3500 (T). Fowler 1928.

Triaenodon obesus (Rüppell, 1837). Fowler and Ball 1925, Myers 1999.
Family Myliobatidae (Eagle Rays)

Aetobatus narinari (Euphrasen, 1790). Myers 1999.

Family Mовulidae (Manta Rays)

Manta birostris (Donndorff, 1798). Myers 1999.

Family Albulidae (Bonefish)

*Albula glossodonta (Forsskål, 1775).

Family Moringuidae (Spaghetti Eels)

*Moringua abbreviata (Bleeker, 1863). вРВм 28145 (G\&R). 
Family Chlop sidae (False Morays)

${ }^{*}$ Kaupicbtbys sp. CAS 60094.

Family Muraenidae (Moray Eels)

*Anarchias sp. врвм 12710 (G\&R).

*Anarchias cantonensis (Schultz, 1943). CAS 149959.

*Anarchias seychellensis Smith, 1962. CAS 51023.

*Echidna leucotaenia Schultz, 1943. cas 28698.

*Gymnomuraena zebra (Shaw in Shaw and Nodder, 1797). Collected by G\&R and kept in the University of Hawai' $i$ fish collection, UH 8-IV-53.

Gymnothorax buroensis (Bleeker, 1857). вРвм 12360 (G\&R). Böhlke and Randall 2000.

*Gymnothorax enigmaticus McCosker \& Randall, 1982. CAs 51024.

Gymnothorax flavimarginatus (Rüppell, 1828). CAs 37106.

Lycondontis flavomarginata (Rüppell). Fowler and Ball 1925 (врвм specimen not located).

Gymnotborax javanicus (Bleeker, 1859). вРвм 12358 (G\&R). Myers 1999, Böhlke and Randall 2000.

*Gymnothorax meleagris (Shaw in Shaw and Nodder, 1795). UH Collection.

Gymnothorax pictus (Ahl, 1789). вРвм 3564, 3565 (T), врвм 12530, 12544 (G\&R). Böhlke and Randall 2000.

Lycodontis picta (Ahl). Fowler and Ball 1925.

Gymnothorax ruppelliae (McClelland, 1845). врвм 3593 (T), врвм 12371, 12527 (G\&R). Lycodontis petelli (Bleeker). Fowler and Ball 1925.

Gymnothorax undulatus (Lacepède, 1803). врвм 12380 (G\&R). Böhlke and Randall 2000.

Lycodontis undulata (Lacepède). Fowler and Ball 1925.

Uropterygius macrocephalus (Bleeker, 1865). CAS 41192. McCosker et al. 1984, Böhlke and Randall 2000.

*Uropterygius xanthopterus Bleeker, 1859. CAS 149964.

Family Opнichthidae (Snake Eels)

*Myricbthys colubrinus (Boddaert, 1781). вРвм 12518 (G\&R).
Myricbthys maculosus (Cuvier, 1817). McCosker and Rosenblatt 1993, Myers 1999.

*Scolecenchelys gymnota (Bleeker, 1857). CAS 200128.

Family Congridae

(Conger and Garden Eels)

${ }^{*}$ Conger cinereus Rüppell, 1830. врвм 12668 (G\&R).

*Heteroconger bassi (Klausewitz \& Eibl Eibesfeldt, 1959). Photographic record.

Family Chanidae (Milkfish)

Chanos chanos (Forsskål, 1775). Myers 1999.

Family Myсторнidde (Lanternfishes)

Myctophum spinosum (Steindachner, 1867). врвм 3643 (T).

Dasyscopelus spinosus (Schneider). Fowler and Ball 1925.

Family Bythitidae (Livebearing Brotulas)

*Dinematichtbys ilucoeteoides Bleeker, 1855. врвм 15169 (G\&R). This fish is probably not ilucoeteoides (J. Randall, pers. comm.), but it has not yet been reclassified.

Family Mugilidae (Mullets)

${ }^{*}$ Crenimugil crenilabis (Forsskål, 1775). врвм 3816 (T). Omitted by Fowler and Ball 1925.

${ }^{*}$ Liza vaigiensis (Quoy \& Gaimard, 1825). A photograph was taken of a juvenile seen in the small boat harbor. This distinctive species has black pectoral fins and yellow pelvic and anal fins.

*Neomyxus leuciscus (Günther, 1872). CAs 150042.

\section{Family Clupeidae (Herrings)}

Spratelloides sp. An unidentified clupeid that may be Spratelloides was photographed in the channel between Wake and Peale Islands.

Family Exocoetidae (Flyingfishes)

${ }^{*}$ Cypselurus poecilopterus (Valenciennes, 1847). в Рвм 10536.

Exocoetus volitans Linnaeus, 1758. врвм 3668, 3669 (T). Fowler and Ball 1925. 
Hirundichtbys rondeletii (Valenciennes, 1847). врвм 3673 (T).

Cypselurus rondelitii (Valenciennes). Fowler and Ball 1925.

Family Hemiramphidae (Halfbeaks)

Hyporbamphus acutus acutus (Gunther, 1872). MCZ 158366. Collette 1974.

Oxyporbampus micropterus (Valenciennes, 1846). Myers 1999.

Family Holocentridae

(Soldierfishes and Squirrelfishes)

*Myripristis adusta (Bleeker, 1853). Photographic record.

Myripristis amaena (Castelnau, 1873). врвм 15173 (G\&R). Randall and Greenfield 1996.

Myripristis berndti Jordan \& Evermann, 1903. врвм 3758 (T), врвм 28241. Randall and Greenfield 1996, Myers 1999. Identified as Myripristis murdjan (Forsskål) in Fowler and Ball 1925.

*Myripristis kuntee Cuvier in $\mathrm{C}$ and $\mathrm{V}$, 1831.

Myripristis murdjan (Forsskål, 1775). Myers 1999 tentatively listed this species.

*Myripristis violacea (Bleeker, 1851). Photographic record.

Neoniphon opercularis (Valenciennes, 1831). врвм 3752 (T).

Holocentrus opercularis Valenciennes.

Fowler and Ball 1925.

Neoniphon sammara (Forsskål, 1775). вРвм 3751 (T), врвм 15110 (G\&R).

Holocentrus sammara Forsskål. Fowler and Ball 1925.

Sargocentron melanospilos (Bleeker, 1858). Myers 1999.

Sargocentron microstoma (Günther, 1859). врвм 3733 (T). Myers 1999.

Holocentrus microstomus Günther. Fowler and Ball 1925.

Sargocentron punctatissimum (Cuvier, 1829). врвм 3715 (T). Myers 1999.

Holocentrus lacteoguttatus (Cuvier). Fowler and Ball 1925.

Sargocentron spiniferum (Forsskål, 1775). вРвм 3738 (T), врвм 15375 (G\&R). Myers 1999. Holocentrus spinifer Forsskål. Fowler and Ball 1925.
Sargocentron tiere (Cuvier in C and V, 1829). ANSP 102854. Randall 1998a, Myers 1999.

Family Sygnathidae

(Pipefishes and Seahorses)

Corythoichthys flavofasciatus (Rüppell, 1838). врвм 3775 (T), врвм 37884 (K\&L). Myers 1999.

Corythoichthys conspicillatus (Jenyns). Fowler and Ball 1925.

Doryrhampus excisus Kaup, 1856. Myers 1999. Family Aulostomidae (Trumpetfishes)

Aulostomus chinensis (Linnaeus, 1766). Fowler and Ball 1925. Although A. chinensis is listed in Fowler and Ball (1925), we did not observe any. Apparently the only Fowler and Ball specimen of $A$. chinensis to survive to the 1960 s was from Lisianski Island. This specimen was discarded by врвм in 1968. Thus this record from Wake Atoll is doubtful.

\section{Family Fistulariddae (Cornetfishes)}

*Fistularia commersonii Rüppell, 1838. Photographic record.

Family Scorpaenidae (Scorpionfishes)

Pterois antennata (Bloch, 1787). вРвм 15280 (G\&R). Myers 1999.

*Scorpaenodes guamensis (Quoy \& Gaimard, 1824). CAs 150161.

Sebastapistes ballieui (Sauvage in Vaillant and Sauvage, 1875). врвм 4365. Identified as Sebastapistes asperella (Bennett) in Fowler and Ball 1925, a species with inadequate description and no type specimen (Eschmeyer and Randall 1975). The врвм lot contains two specimens, one $S$. ballieui and one $S$. cyanostigma; however, the locality of this lot was incorrectly listed as Wake (J. Randall, pers. comm.). This species is not included in the species count.

Sebastapistes coniorta Jenkins, 1903. Randall 1996, Myers 1999.

*Sebastapistes fowleri (Pietschmann, 1934). врвм 38593 (K\&L).

* Sepastapistes mauritiana (Cuvier, 1829). врвм 12043 (K), врвм 13804 (G\&R).

*Sebastapistes tinkbami (Fowler, 1946). MCZ 158370. 
Family Caracanthidae

(Orbiculate Velvetfishes)

*Caracantbus maculatus (Gray, 1831). вРвм 4422 (T), MCZ 158364. This record was omitted from Fowler and Ball 1925.

Caracantbus unipinna (Gray, 1831). врвм 4425 (T). Fowler and Ball 1925.

\section{Family Serranidae \\ (Groupers and Sea Basses)}

*Aporops bilinearis Schultz, 1943. cas 82471.

Cephalopholis argus (Schneider, 1801). врвм 3951 (T), врвм 12042 (K), вРвм 15098, 15144 (G\&R), su 500065, ANSP 88339. Fowler and Ball 1925, Randall and Heemstra 1991, Myers 1999.

Cephalopholis spiloparaea (Valenciennes, 1828). врвм 3950 (T). Randall and Heemstra 1991, Myers 1999. This specimen was misidentified as Cephalopholis sonnerati (Valenciennes) by Fowler and Ball 1925.

${ }^{*}$ Cephalopholis urodeta (Forster, 1801). Photographic record.

Epinephelus fasciatus (Forsskål, 1775). ANSP 91775, 91783, врвм 3956 (Т), врвм 15428 (G\&R). Randall and Heemstra 1991, Myers 1999.

Holocentrus erythraeus (Bloch \& Schneider). Fowler and Ball 1925.

Serranus fasciatus (Forsskål). Fowler and Ball 1925.

Epinephelus bexagonatus (Forster, 1801). ANSP 102856, вРвм 3976 (T) 12040. Randall and Heemstra 1991, Myers 1999. Specimen врвм 3976 was misidentified as Serranus merra by Fowler and Ball 1925.

Epinephelus lanceolatus (Bloch, 1790). Myers 1999.

*Epinephelus merra Bloch, 1790. вРвм 12040 (G\&R).

Epinephelus polyphekadion (Bleeker, 1849). Randall and Heemstra 1991, Myers 1999.

Epinephelus spilotoceps Schultz, 1953. Myers 1999.

Epinephelus tauvina (Forsskål, 1775). ANSP 105506, врвм 3963 (T). Randall and Heemstra 1991, Myers 1999. This specimen was misidentified as Serranus corallicola (Valenciennes) by Fowler and Ball 1925.
*Liopropoma tonstrinum Randall \& Taylor, 1988. врвм 37889 (K\&L).

*Plectrantbias longimanus (Weber, 1913). MCZ 158369.

${ }^{*}$ Plectranthias nanus Randall, 1980. MCZ 158368.

${ }^{*}$ Plectranthias winniensis (Tyler, 1966). вРвм 37890 (K\&L).

Pseudantbias pascalus (Jordan \& Tanaka, 1927). врвм 37891 (K\&L). Myers 1999.

*Pseudanthias ventralis (Randall, 1979). врвм 37892 (K\&L).

*Pseudogramma polyacantha (Bleeker, 1856). врвм 37893 (K\&L).

Variola louti (Forsskål, 1775). врвм 39473948 (T). Fowler and Ball 1925, Randall and Heemstra 1991, Myers 1999.

Family Cirrhitidae (Hawkfishes)

*Amblycirrbitus bimacula (Jenkins, 1903). CAS 24409.

Cirrbitus pinnulatus (Schneider in Bloch and Schneider, 1801). врвм 4103 (T), врвм 15121 (G\&R).

Cirrbitus maculatus (Lacepède, 1803). Fowler and Ball 1925.

Neocirrbites armatus Castlenau, 1873. вРвм 4122 (T). Myers 1999.

Paracirrbites melanotus (Günther). Fowler and Ball 1925.

Paracirrbites arcatus (Cuvier in C and V, 1829). Myers 1999.

Paracirrbites forsteri (Schneider in Bloch and Schneider, 1801). врвм 4126 (T). Myers 1999.

Paracirrbites bemistictus (Günther, 1874). Myers 1999.

Family Pseudochromidae (Dottybacks)

*Pseudochromis sp. Video taken on the eastern side of Wake Island of a pseudochromid that is likely to be either $P$. fuscus or $P$. cyanotaenia. R. F. Myers (pers. comm.) noted that this is likely to be $P$. cyanotaenia, not $P$. fuscus.

*Pseudoplesiops sp. Specimen collected in 1997. This is an unidentified species.

Family Priacanthidae (Bigeyes, Glasseyes)

*Heteropriacanthus cruentatus (Lacepède, 1801). Photographic record. 
Family Apogonidae (Cardinalfishes)

*Apogon coccineus Ruppell, 1838. cas 150060. *Apogon cyanosoma Bleeker, 1853. cas 85030. Apogon doryssa (Jordan \& Seale, 1906). врвм $3901(\mathrm{~T})$.

Amia doryssa (Jordan \& Seale). Fowler and Ball 1925. Specimen could not be located.

Apogon exostigma (Jordan \& Starks, 1906). Myers 1999.

*Apogon fuscus Quoy \& Gaimard, 1825. Photograph was tentatively identified as $A$. fuscus.

Apogon kallopterus Bleeker, 1856. вРвм 15273 (G\&R), врвм 37876 (K\&L). Myers 1999.

*Apogon savayensis (Günther, 1871). вРвм 3894 (T), врвм 12041 (K), мСZ 158363. Probably misidentified as Amia (Apogon) bandanensis (Bleeker) by Fowler and Ball 1925.

Apogon susanae Greenfield, 2001. cas 84823. This specimen from Wake was misidentified as $A$. coccineus. The new species $A$. susanae described by Greenfield (2001) included this specimen in the description.

*Apogon taeniophorus Regan, 1905.

*Cheilodipterus macrodon (Lacepède, 1802). MCZ 158371.

*Cheilodipterus quinquelineata (Cuvier, 1828). врвм 37877 (K\&L).

${ }^{*}$ Fowleria isostigma (Jordan \& Seale, 1906). врвм 3916 (T). Identified as Apogonichthys auritus (Valenciennes) (current name Fowleria aurita) by Fowler and Ball 1925.

Family Malacanthidae (Sand Tilefishes)

*Malacanthus brevirostris Guichenot, 1848.

Family Echeneidae (Remoras)

Remora remora (Linnaeus, 1758). вРвм 4894 (T).

Echeneis remora (Linnaeus). Fowler and Ball 1925.

${ }^{*}$ Remora osteochir (Cuvier, 1829). usNm 202330, 202332. Exclusively pelagic species collected from the National Marine Fisheries Service R.V. Charles H. Gilbert and placed in the USNM by D. W. Strasburg (U.S. Fish and Wildlife Service).
Family Cory phaenidae (Dolphinfishes)

Coryphaena bippurus Linnaeus, 1758. Myers 1999.

Family Carangidae (Jacks)

Carangoides ferdau (Forsskål, 1775). Fowler and Ball 1925.

${ }^{*}$ Carangoides orthogrammus (Jordan \& Gilbert, 1881). врвм 3885 (T). Photographic record.

*Caranx ignobilis (Forsskål, 1775). Photographic record.

Caranx lugubris Poey, 1861. Myers 1999.

Caranx melampygus Cuvier in C and V, 1833. Myers 1999.

Caranx sexfasciatus Quoy \& Gaimard, 1824. Myers 1999.

*Decapturus macarellus (Cuvier, 1833). Photographic record.

*Elagatis bipinnulatas (Quoy \& Gaimard, 1824). Species identified from a photograph taken by local fishermen.

*Gnathanodon speciosus (Forsskål, 1775). Photographic record.

Scomberoides lysan (Forsskål, 1775). Myers 1999.

Seriola rivoliana Valenciennes, 1833. Myers 1999.

*Tracbinotus baillonii (Lacepède, 1801). cAs 24035.

Family Lutjanidae (Snappers)

*Aphareus furca (Lacepède, 1801). Photographic record.

*Aprion virescens Valenciennes, 1830. Photographic record.

Lutjanus fulvus (Schneider, 1801). врвм 4006, 4007. Myers 1999.

Lutjanus marginatus (Cuvier). Fowler and Ball 1925.

Lutjanus monostigma (Cuvier, 1828). вРвм 4012 (T).

Lutjanus monostigma (Cuvier). Fowler and Ball 1925.

Family Lethrinidae (Emperors)

Letbrinus obsoletus (Forsskål, 1775). Myers 1999.

${ }^{*}$ Letbrinus rubrioperculatus Sato, 1978. врвм 4023 (T). Originally identified as Letbri- 
nus mabsena (Forsskål) by Fowler and Ball 1925.

Monotaxis grandoculis (Forsskål, 1775). вРвм 4032 (T). Fowler and Ball 1925, Myers 1999.

\section{Family Mullidae (Goatfishes)}

*Mulloidicbthys flavolineatus (Lacepède, 1801). врвм 4089 ( $\mathrm{T})$.

*Mulloidichthys vanicolensis (Valenciennes in $\mathrm{C}$ and V, 1831). Photographic record.

Parupeneus barberinus (Lacepède, 1801). Myers 1999.

Parupeneus cyclostomus (Lacepède, 1801). врвм 4077 (T). Myers 1999.

Upeneus chryserydros (Lacepède). Fowler and Ball 1925.

Parupeneus insularis Randall \& Myers, 2002. врвм 4070 (T), врвм 15310, 15442 (G\&R). Specimen врвм 4070 was identified by Fowler and Ball 1925 as Upeneus indicus (Shaw). Until recently, this species was classified as $P$. bifasciatus in the central Pacific (Randall and Myers 2002).

Parupeneus multifasciatus (Quoy \& Gaimard, 1825). Myers 1999.

*Parupeneus pleurostigma (Bennett, 1830).

Upeneus arge Jordan \& Evermann, 1903. Myers 1999.

Family Pempheridae (Sweepers)

${ }^{*}$ Pempheris oualensis Cuvier, 1831. Photographic record.

Family Chaetodontidae (Butterflyfishes)

Chaetodon auriga Forsskål, 1775. врвм 4183. Myers 1999.

Chaetodon setifer Bloch. Fowler and Ball 1925.

Chaetodon ephippium Cuvier in C and V, 1831. врвм 4149 (T), врвм 15183 (G\&R). Fowler and Ball 1925, Myers 1999.

*Chaetodon lineolatus Cuvier in C and V, 1831. Video was taken of this species.

Chaetodon lunula (Lacepède, 1803). врвм 4164 (T), врвм 15193 (G\&R). Fowler and Ball 1925, Myers 1999.

Chaetodon ornatissimus Cuvier in C and V, 1831. Fowler and Ball 1925, Myers 1999.

Chaetodon punctatofasciatus Cuvier, 1831. Myers 1999.
Chaetodon quadrimaculatus Gray, 1831. врвм 15192 (G\&R). Fowler and Ball 1925, Myers 1999.

Chaetodon reticulatus Cuvier in C and V, 1831. врвм 4167 ( $\mathrm{T})$. This specimen was misidentified as Chaetodon collaris Bloch by Fowler and Ball 1925. Also reported by Myers 1999.

Chaetodon semeion Bleeker, 1855. врвм 4150 (T). Fowler and Ball 1925.

Chaetodon ulietensis Cuvier, 1831. врвм 4195, 4196 (T). Myers 1999. Misidentified as Chaetodon falcula Bloch in Fowler and Ball 1925.

${ }^{*}$ Chaetodon unimaculatus Bloch, 1787. CAS 150086.

*Forcipiger flavissimus Jordan \& McGregor, 1898. Photographic record.

Forcipiger longirostris (Broussonet, 1782). Fowler and Ball 1925, Myers 1999.

Hemitaurichtbys thompsoni Fowler, 1923. Myers 1999.

Heniochus acuminatus (Linnaeus, 1758). Myers 1999.

Family Pomacanthidae (Angelfishes)

Centropyge flavissima (Cuvier, 1831). врвм 4221 (T), врвм 15344 (G\&R). Myers 1999.

Holocanthus flavissimus (Cuvier). Fowler and Ball 1925.

Centropyge loricula (Günther, 1873). Myers 1999.

*Centropyge multicolor Randall \& Wass, 1974. Photographic record.

\section{Family Ky phosidae \\ (Rudderfishes, Sea Chubs)}

Kyphosus bigibbus Lacepède, 1802. врвм 4043, 4044 (T). Myers 1999.

Kyphosus fuscus (Quoy \& Gaimard). врвм 4043 (T). Omitted by Fowler and Ball 1925.

Kyphosus cinerascens (Forsskål, 1775). Myers 1999.

\section{Family Kunliddae (Flagtails)}

Kublia sandvicensis (Steindachner, 1876). врвм 3929 (T). Fowler and Ball 1925. These specimens were determined as the true sandvicensis (which ranges beyond Hawai' $\mathrm{i}$ ), 
although some characters (large eye and some meristic data) do not fit well. The possibility exists that this could be some other species (Randall and Randall 2001).

\section{Family Oplegnathidae (Knifejaws)}

*Oplegnathus punctatus (Temminck \& Schlegel, 1844). врвм 37896 (K\&L).

Family Pomacentridae (Damselfishes)

Abudefduf septemfasciatus (Cuvier, 1830). вРвм 4506 (T). Fowler and Ball 1925.

Abudefduf sordidus (Forsskål, 1775). вРвм 4490, 4491 (T). Fowler and Ball 1925.

*Abudefduf vaigiensis (Linnaeus, 1758). Photographic record.

Chromis acares Randall \& Swerdloff, 1973. врвм 4450 (T), MCZ 158367. Myers 1999. Identified as Cbromis dimidiatus (Klunzinger) by Fowler and Ball 1925 .

Chromis agilis Smith, 1960. вРвм 37888 (K\&L). Myers 1999.

${ }^{*}$ Chromis vanderbilti (Fowler, 1941). врвм 12300 (G\&R). Photographic record.

Chromis viridis (Cuvier, 1830). Specimens listed as Chromis caeruleus (Cuvier) in Fowler and Ball 1925 and as C. caerulea, врвм 4454 (T), врвм 15319 (G\&R). Cbromis caerulea has been suppressed. Chromis ternatensis, which formerly had been synonymized with $C$. caerulea (Allen 1991), was validated as a distinct species and the next available name for what had been called $C$. caerulea was $C$. viridis (Randall et al. 1987).

Chrysiptera biocellata (Quoy \& Gaimard, 1824). врвм 4516 (T). Identified as $A b u$ defduf brownriggii (Bennett) by Fowler and Ball 1925.

Chrysiptera brownriggii (Bennett, 1828). ВРВМ 4528, 4540 (T).

Abudefduf amabilis (De Vis). Fowler and Ball 1925.

Abudefduf leucopomus (Cuvier). Fowler and Ball 1925.

${ }^{*}$ Cbrysiptera glauca (Cuvier, 1830). врвм 4542

(T), врвм 15362 (G\&R). Identified as Abudefduf zonatus (Cuvier) by Fowler and Ball 1925.
Dascyllus aruanus (Linnaeus, 1758). ВРвм 4443 (T), врвм 15178, 15367 (G\&R). Fowler and Ball 1925.

Plectroglyphidodon dickii (Liénard, 1839). Myers 1999.

Plectroglyphidodon imparipennis (Vaillant \& Sauvage, 1875). врвм 4536 (T), врвм 15341 (G\&R).

Abudefduf imparipennis (Vaillant \& Sauvage). Fowler and Ball 1925.

Plectroglyphidodon jobnstonianus Fowler \& Ball, 1924. Myers 1999.

*Plectroglyphidodon lacrymatus (Quoy \& Gaimard, 1825). Photographic record.

*Plectroglyphidodon phoenixensis (Schultz, 1943). UH collection.

Stegastes albifasciatus (Schlegel \& Müller, 1839). No specimens found in the врвм. Abudefduf albofasciatus (Schlegel \& Müller). Fowler and Ball 1925.

*Stegastes fasciolatus (Ogilby, 1889). Photographic record.

Stegastes nigricans (Lacepède, 1803). врвм 4464, 4465 (T), врвм 15177 (G\&R).

Pomacentrus nigricans (Lacepède). Fowler and Ball 1925.

\section{Family Labridae (Wrasses)}

*Ammolabrus dicrus Randall \& Carlson, 1997. врвм 38592 (K\&L). This species is currently known only from $\mathrm{O}^{6} \mathrm{ahu}$ and Wake Atoll. The same species or a close relative was observed in the Ogasawara Islands, Japan, but was not collected (Randall and Carlson 1997).

Anampses caeruleopunctatus Rüppell, 1829. MCZ 158361. Myers 1999.

*Bodianus antbioides (Bennett, 1831). Photographic record.

*Cheilinus chlorourus (Bloch, 1791). вРвм 4688 (T), врвм 12047 (K), врвм 12723 (G\&R) Originally identified as Cheilinus undulatus Rüppell by Fowler and Ball 1925.

${ }^{*}$ Cheilinus fasciatus (Bloch, 1791).

Cheilinus trilobatus Lacepède, 1801. Myers 1999.

Cheilinus undulatus Rüppell, 1835. Myers 1999.

Coris aygula Lacepède, 1801. врвм 5116, 12046 (K). Fowler and Ball 1925, Randall 1999. 
Epibulis insidiator (Pallas, 1770). вРвм 12044 (K). Myers 1999.

Gomphosus varius Lacepède, 1801. вРвм 12045 (K). Myers 1999.

Halichoeres biocellatus Schultz, 1960. Myers 1999.

${ }^{*}$ Halichoeres margaritaceus (Valenciennes in C and V, 1839). CAs 73712.

${ }^{*}$ Halichoeres ornatissimus (Garrett, 1863). вРвм 15347 (G\&R).

Halichoeres trimaculatus (Quoy \& Gaimard, 1824). врвм 4577 (T), врвм 12712, 12715, 15172 (G\&R). Fowler and Ball 1925.

Hemigymnus fasciatus (Bloch, 1792). Myers 1999.

*Iniistius sp. An unknown species with an allwhite body was observed in the lagoon on several occasions. No specimens were collected.

Labroides bicolor Fowler \& Bean, 1928. Myers 1999.

Labroides dimidiatus (Valenciennes, 1839). Myers 1999.

Labroides pectoralis Randall \& Springer, 1975. врвм 37886 (K\&L). Myers 1999.

*Labroides rubrolabiatus Randall, 1958. CAS 73544.

Novaculicbthys taeniouris (Lacepède, 1801). врвм 4696 (T). Fowler and Ball 1925.

Oxycheilinus diagrammus (Lacepède, 1801). Cheilinus diagrammus (Lacepède). Fowler and Ball 1925.

${ }^{*}$ Oxycheilinus orientalis (Günther, 1862).

*Oxycheilinus unifasciatus (Streets, 1877). вРвм 12722 (G\&R).

Pseudodcheilinus bexataenia (Bleeker, 1857). врвм 4686 (T). Fowler and Ball 1925.

*Pseudocheilinus ocellatus Randall, 1999. врвм 37887, paratype (K\&L).

*Pseudocheilinus octotaenia Jenkins, 1900.

*Pseudocheilinus tetrataenia Schultz, 1960.

*Pseudocoris aurantiofasciata Fourmanoir, 1971. врвм 38772. These fish were observed feeding in mixed schools with Acanthurus thompsoni in 18 to $20 \mathrm{~m}$ near the wreck of the Stoner.

Pseudojuloides atavai Randall \& Randall, 1981. Myers 1999.

*Pseudojuloides cerasinus (Snyder, 1904). Photographic record.
Stethojulis bandanensis (Bleeker, 1851). вРвм 12730, 12732, 15205 (G\&R). Myers 1999.

*Thalassoma bardwicke (Bennett, 1820). CAS 75547.

Thalassoma lutescens (Lay \& Bennett, 1839). вРвм 4646 ( $\mathrm{T})$, врвм 12680, 12687 (G\&R). Myers 1999.

Thalassoma purpureum (Forsskål, 1775). вРвм 4640, 4641 (T). Fowler and Ball 1925. Thalassoma umbrostigma (Rüppell). Fowler and Ball 1925 also spelled it umbrostygma.

Thalassoma quinquevittatum (Lay \& Bennett, 1839). врвм 4637 (T), врвм 15244 (G\&R). Myers 1999. Misidentified as Thalassoma guntheri (Bleeker) by Fowler and Ball 1925.

*Thalassoma trilobatum (Lacepède, 1801). врвм 15202 (G\&R).

*Xyrichtys undescribed species. врвм 38591, paratype $(K \& L)$. J. Earle and B. Halstead collected one pair of this species from New Guinea in 1993 and it was photographed in Guam by T. Allen. We collected specimens from Wake at $20 \mathrm{~m}$ over sand, directly in front of the small boat harbor. For description of this species see Randall and Lobel (in press).

\section{Family Scaridae (Parrotfishes)}

Bolbometopon muricatum (Valenciennes, 1839). Myers 1999.

Callyodon borborus Jordan \& Evermann, 1903 reported by Fowler and Ball 1925 is a synonym of the Hawaiian endemic Chlorurus perspicillatus (Steindachner). No specimens were found at врвм. This species is not included in the species count.

Chlorurus frontalis Valenciennes, 1839. Myers 1999.

Callyodon jonesi (Streets). Fowler and Ball 1925.

Chlorurus microrbinos (Bleeker, 1854). Myers 1999.

Cblorurus sordidus (Forsskål, 1775). вРвм 4739, 4748 (T).

Callyodon sordidus (Forsskål). Fowler and Ball 1925.

Callyodon erytbrodon (Valenciennes). Fowler and Ball 1925.

${ }^{*}$ Hipposcarus longiceps (Valenciennes, 1839). Photographic record. 
Scarus forsteni (Bleeker, 1861). врвм 4770 (T). Myers 1999. Misidentified as Callyodon (Scarus) tricolor (Bleeker) by Fowler and Ball 1925.

*Scarus ghobban Forsskål, 1775.

${ }^{*}$ Scarus globiceps Valenciennes, 1840.

Scarus oviceps Valenciennes, 1840. врвм 4766 (T). Myers 1999. Callyodon pectoralis (Valenciennes) reported by Fowler and Ball 1925 is a synonym of Scarus scaber Valenciennes, an Indian Ocean species. No specimens were found at врвм. This was probably a misidentification of $S$. oviceps.

Scarus psittacus Forsskål, 1775. вРвм 4749, 4753 (T).

Callyodon brunneus (Jenkins). Fowler and Ball 1925.

Scarus rubroviolaceus Bleeker, 1849. врвм 4773 (T).

Callyodon rubroviolaceus (Steindachner). Fowler and Ball 1925.

Family Pinguipedidae (Sandperches)

*Parapercis schauinslandii (Steindachner, 1900).

Family Creedidae (Sand Burrowers)

*Limnichthys nitidus Smith, 1958. mCz 158368. Originally identified as $L$. donaldsoni Schultz, 1960, which has been shown to be a synonym of nitidus (Yoshino et al. 1999).

Family Tripterygidae (Triplefins)

${ }^{*}$ Enneapterygius nigricauda Fricke, 1997. врвм 4910 (T). This specimen was identified as Enneapterygius bemimelas (Kner \& Steindachner, 1866) by Fowler and Ball 1925.

*Helcogramma cbica Rosenblatt in Schultz, 1960. врвм 37885 (K\&L).

\section{Family BlenNiddae (Blennies)}

Blenniella gibbifrons (Quoy \& Gaimard, 1824). Istiblennius gibbifrons (Quoy \& Gaimard). врвм 15364, 15378 (G\&R). Springer and Williams 1990.

Rupiscartes gibbifrons (Quoy \& Gaimard). Fowler and Ball 1925.

Blenniella paula (Bryan \& Herre, 1903). врвм 4938 (T), врвм 15368 (G\&R). Springer and Williams 1994. A specimen listed in the CAS (150165) as Blenniella periopbthalmus (Valenciennes, 1836) is probably B. paula.
Cirripectes polyzona (Bleeker, 1868). Myers 1999.

Cirripectes quagga (Fowler \& Ball, 1924). ANSP 91191, 91013, вРвм 3420 (Т), вРвм 30998. Williams 1988.

Rupiscartes quagga Fowler \& Ball. врвм 3420, 10436 (T). Fowler and Ball 1925.

Cirripectes variolosus (Valenciennes, 1836). врвм 4925 (T), врвм 15369 (G\&R).

Rupiscartes variolosus (Valenciennes). Fowler and Ball 1925.

*Entomacrodus marmoratus (Bennett, 1828). врвм 4933 (T). This specimen is listed in the врвм records as having been collected by the Tanager Expedition at Wake Atoll, but the specimen is missing (A. Suzumoto, pers. comm.). This is a dubious record because E. marmoratus is a Hawaiian endemic and needs to be confirmed by additional collections.

Entomacrodus striatus (Quoy \& Gaimard, 1836). вРвм 12060, 14210 (G\&R). Myers 1999.

Istiblennius edentulus (Bloch \& Schneider, 1801). вРвм 4944 (T), вРвм 15209 (G\&R), MCz 158366. Myers 1999.

Salarias edentulus (Schneider). Fowler and Ball 1925.

*Plagiotremus tapienosoma (Bleeker, 1857). CAS 29079.

Rhabdoblennius ellipes Jordan \& Starks, 1906. Blennius rbabdotrachelus Fowler \& Ball. врвм 3419 (T). Fowler and Ball 1925.

\section{Family Callionymidae (Dragonets)}

*Synchiropus laddi Schultz in Schultz et al., 1960. врвм 38302 (G\&R). Specimen originally labeled as Synchiropus kiyoae Fricke \& Zaiser was reidentified as $S$. laddi by J. Randall.

*Callionymidae unidentified species. MCZ 158373.

$$
\text { Family Gов indae (Gobies) }
$$

*Amblygobius phalaena (Valenciennes, 1837). врвм 37878 (K\&L).

*Asterropteryx semipunctatus Rüppell, 1830. врвм 37879 (K\&L).

Batbygobius fuscus (Rüppell, 1828). вРвм 4866 (T). Fowler and Ball 1925.

${ }^{*}$ Cabillus tongarevae (Fowler, 1927). UsNM 
166748 (G\&R 1953). The identity of this specimen needs to be reexamined because its occurrence at Wake is doubtful ( $\mathrm{J}$. Randall, pers. comm.).

${ }^{*}$ Coryphopterus duospilus (Hoese \& Reader, 1985). врвм 37881 (K\&L).

${ }^{*}$ Coryphopterus neopbytus (Günther, 1877). врвм 37880 (K\&L).

Coryphopterus sp. врвм 4844 (T). This specimen collected in salt water was identified as the brackish-water Glossogobius giuris (Hamilton) by Fowler and Ball 1925.

${ }^{*}$ Ctenogobiops aurocingulus (Herre, 1935). USNM 166766 (G\&R).

${ }^{*}$ Ctenogobiops feroculus Lubbock \& Pulonin, 1977. Photographic record. This species was identified from a photograph; however, it is possible that it is another species (J. Randall, pers. comm.).

Ctenogobiops pomastictus Lubbock \& Pulonin, 1977. Tentatively listed in Myers 1999.

Eviota alfelei Jordan \& Seale, 1906. врвм 15343, 15166 (G\&R), врвм 37882 (K\&L). Lachner and Karnella 1980.

*Eviota epiphanes Jenkins, 1903. врвм 4816 (T). This specimen is listed in the врвм records as having been collected by the Tanager Expedition at Wake Atoll, but the specimen is missing (A. Suzumoto, pers. comm.). Identified as E. viridis (Waite) by Fowler and Ball 1925. The identification of this specimen as E. epiphanes is tentative. Lachner and Karnella (1980) remarked that this species is restricted to the North Central Pacific and is found the entire length from Midway to Johnston to Kirimati (Christmas Island); however, Karnella and Lachner (1981) also noted a tentative identification of $E$. epiphanes at Minami-tori-shima (Marcus) Island. Because other Hawaiian endemics are potentially found at Wake, there is a possibility that this species is as well.

*Eviota saipanensis Fowler, 1945. врвм 15187 (G\&R).

${ }^{*}$ Favonigobius sp. Specimen collected by us in 1997 is an undescribed species.

Gnatholepis cauerensis cauerensis (Bleeker, 1853).

Gnatholepis anjerensis (Bleeker). врвм 4870 (T). Fowler and Ball 1925.
Gobiodon rivulatus (Rüppell, 1830). вРвм 4823 (T). Fowler and Ball 1925.

*Paragobiodon lacunicolus (Kendall \& Goldsborough, 1911). вРвм 4834 (T). Originally identified as Paragobiodon echinocephalus (Rüppell) by Fowler and Ball 1925 .

*Priolepis kappa Winterbottom \& Burridge, 1993. Cas 60011.

${ }^{*}$ Priolepis semidoliatus (Valenciennes, 1837). usNM 166779 (G\&R).

*Trimma sp. Specimen collected in 1997 may be an undescribed species. Specimens in our collection.

Family Microdesmidae

(Dartfishes and Wormfishes)

*Ptereleotris evides (Jordan \& Hubbs, 1925). Photographic record.

*Ptereleotris microlepis Bleeker, 1856.

Family Siganidae (Rabbitfishes)

*Siganus argenteus (Quoy \& Gaimard, 1825). Photographic record.

Family Zanclidae (Moorish Idol)

Zanclus cornutus (Linnaeus, 1758). Myers 1999.

Family Acanthuridae (Surgeonfishes)

Acanthurus achilles Shaw, 1803. вРвм 4294 (T). Myers 1999.

Hepatus achilles (Shaw). Fowler and Ball 1925.

*Acantburus blocbii Valenciennes in $\mathrm{C}$ and V, 1835. Photographic record.

Acanthurus guttatus Forster, 1801. вРвм 4251

(T). Myers 1999.

Hepatus guttatus (Forster). Fowler and Ball 1925.

Acanthurus leucopareius (Jenkins, 1903). Myers 1999.

Acantburus nigricans (Linnaeus, 1758).

Hepatus nigricans (Linnaeus). Fowler and Ball 1925.

Acanthurus nigricauda Dunker \& Mohr, 1929. врвм 4285 (T). Myers 1999.

Acantburus nigrofuscus (Forsskål, 1775). Myers 1999.

Acanthurus nigroris Valenciennes, 1835. вРвм 4276 (T). Myers 1999. Originally identified as Hepatus elongatus (Lacepède) by Fowler and Ball 1925. 
*Acanthurus nubilus Fowler \& Bean, 1929. врвм 37895 (K\&L).

Acantburus thompsoni (Fowler, 1923). Myers 1999.

Hepatus thompsoni Fowler. Fowler and Ball 1925.

Acanthurus triostegus (Linnaeus, 1758). вРвм 4239, 4278 (T), врвм 15255 (G\&R). Myers 1999.

Hepatus sandvicensis (Streets). Fowler and Ball 1925.

Ctenochaetus cyanocheilus Randall \& Clements, 2001.

${ }^{*}$ Ctenochaetus bawaiiensis Randall, 1955. Photographic record.

Ctenochaetus striatus (Quoy \& Gaimard, 1825). врвм 4302, 4304 (T), сAs 150134. Randall and Clements 2001. Misidentified as $C$. strigosus (Bennett) by Fowler and Ball 1925. Ctenochaetus strigosus is found only in Hawai'i and Johnston Atoll (Randall and Clements 2001).

*Naso brevirostris (Valenciennes, 1835). Photographic record.

*Naso bexacanthus (Bleeker, 1855). Photographic record. This could also be Naso caesius Randall \& Bell. Naso bexacantbus is most common, but $N$. caesius is antitropical and therefore could also occur at Wake (J. Randall, pers. comm.).

Naso lituratus (Bloch \& Schneider, 1801). врвм 4318 (T). Myers 1999.

Acanthurus lituratus (Schneider). Fowler and Ball 1925.

${ }^{*}$ Naso unicornis (Forsskål, 1775). Video and photographs were taken of this species.

*Naso vlamingii (Valenciennes, 1835). Photographic record.

Zebrasoma flavescens (Bennett, 1828). врвм 4312 (T). Myers 1999.

*Zebrasoma veliferum (Bloch, 1797). Photographic record.

Family Sphyraenidae (Barracudas)

Sphyraena barracuda (Walbaum, 1792). Myers 1999.

Family Scomв Ridae (Tunas and Mackerels)

Acanthocybium solanderi (Cuvier in $\mathrm{C}$ and $\mathrm{V}$, 1831). Myers 1999.

Eutbynnus affinis (Cantor, 1849). Myers 1999.
Katsuwonus pelamis (Linnaeus, 1758). Myers 1999.

Tbunnus alalunga (Bonnaterre, 1788). Myers 1999.

Thunnus albacares (Bonnaterre, 1788). Myers 1999.

Thunnus obesus (Lowe, 1839). Myers 1999.

Family Istiophoridae (Billfishes)

Istiophorus platypterus (Shaw \& Nodder, 1792). Myers 1999.

Makaira indica (Cuvier, 1831). Myers 1999.

Makaira mazara (Jordan \& Synder, 1901). Myers 1999. The specimen mounted in the Wake Atoll airport is labeled as $M a$ kaira nigricans Lacepède, 1802, the Atlantic counterpart of $M$. mazara.

Tetrapturus angustirostris Tanaka, 1915. Myers 1999.

Tetrapturus audax (Philippi, 1887). Myers 1999.

\section{Family Nomeidae (Driftfishes)}

Cubiceps pauciradiatus Günther, 1872. Myers 1999.

Family Bothidae (Lefteye Flounders)

Bothus mancus (Broussonet, 1782). вРвм 3687 (T).

Platophrys mancus (Broussonet). Fowler and Ball 1925.

*Bothus pantherinus (Rüppell, 1830). вРвм 15189 (G\&R).

\section{Family Balistidae (Triggerfishes)}

*Balistoides viridescens (Bloch \& Schneider, 1801).

Melichthys niger (Bloch, 1786). врвм 5038 (T). Myers 1999.

Melichtbys buniva (Lacepède). Fowler and Ball 1925.

Melicbthys vidua (Solander in Richardson, 1844). врвм 5004 (T). Myers 1999.

Balistes vidua (Richardson). Fowler and Ball 1925.

Rbinecantbus aculeatus (Linnaeus, 1758). вРвм 5022 (T). Myers 1999.

Balistipus aculeatus (Linnaeus). Fowler and Ball 1925.

${ }^{*}$ Rbinecanthus rectangulus (Bloch \& Schneider, 1801). Photographic record. 
*Sufflamen bursa (Bloch \& Schneider, 1801). Photographic record.

Xantbichthys mento (Jordan \& Gilbert, 1882). Myers 1999.

Family Monacanthidae (Filefishes)

*Aluterus scriptus (Osbeck, 1765). Photographic record.

${ }^{*}$ Cantberbines dumerilii (Hollard, 1854).

Family OstraciIdae

(Trunkfishes, Boxfishes)

Ostracion cubicus Linnaeus, 1758.

Ostracion lentiginosus Bloch \& Schneider. Fowler and Ball 1925.

Ostracion meleagris Shaw, 1796. врвм 12067 (G\&R).

Ostracion sebae Bleeker. Fowler and Ball 1925.

Ostracion camurum Jenkins. врвм 5059, $5064(\mathrm{~T})$.

\section{Family Tetraodontidae (Puffers)}

*Arotbron bispidus (Linnaeus, 1758). Photographic record.

*Arotbron meleagris (Bloch \& Schneider, 1801). врвм 5111, 5117 (T), мСZ 158362. Identified as Tetrodon regani Günther by Fowler and Ball 1925.

${ }^{*}$ Canthigaster amboinensis (Bleeker, 1865). врвм 15358 (G\&R).

Canthigaster janthinoptera (Bleeker, 1855). врвм 37894 (K\&L). Allen and Randall 1977.

${ }^{*}$ Canthigaster solandri (Richardson, 1845). врвм 15332 (G\&R).

Family Diodontidae (Porcupinefishes)

Diodon bystrix Linnaeus, 1758. Fowler and Ball 1925.

\section{DISCUSSION}

The fauna of Wake Atoll is largely composed of wide-ranging western and Indo-Pacific fishes, with the notable occurrence of one Hawaiian endemic species (Table 1). The fish fauna of Wake Atoll is much less diverse than those of the Marshall and Mariana Archipelagos. This is probably due to the isolation and low diversity of habitat types found on
TABLE 1

Zoogeography of Wake Atoll Fishes

\begin{tabular}{lc}
\hline \hline $\begin{array}{l}\text { Zoogeographic } \\
\text { Distribution }\end{array}$ & $\begin{array}{c}\text { \% of Wake } \\
\text { Atoll Fishes }\end{array}$ \\
\hline Hawai'i/Japan & 0.3 \\
Indo-Pacific & 52.2 \\
Indo-pan-Pacific & 11.1 \\
West central Pacific & 11.1 \\
Indo-West Pacific & 3.2 \\
West Pacific & 1.3 \\
Pacific Plate & 5.7 \\
Pan-Pacific & 1.0 \\
Circumtropical & 4.1 \\
Circumglobal & 1.3 \\
\hline
\end{tabular}

the atoll. The Mariana Islands are composed of atolls and high islands with diverse habitat types. The Marshall Islands contain atolls with habitats similar to those of Wake but covering a much larger area and therefore benefiting from increased connectivity between reef systems. In comparison, Wake is an isolated single atoll with relatively few habitat types.

The geomorphology of the atoll is shown in Figures 2 and 3. There are four basic aquatic habitat types within Wake Atoll. The first includes a shallow and turbid lagoon with scattered patch reefs. About one-fourth of the lagoon habitat consists of featureless sand flats that are fully exposed at low tide. A second habitat type is the intertidal, hard-substrate, ocean reef flat, also exposed at low tide. The third is the reef crest, of which the top is also exposed at low tide. The fourth habitat type is the outer reef, which drops in depth rapidly, on both the exposed windward and sheltered leeward sides. We did not find sea grass, algal flats, or mangroves. There are no brackishwater habitats. The island's topography is very low, with a maximum height above sea level of only a few meters.

The lagoon is very murky and shallow (maximum depth of about $5 \mathrm{~m}$ ). The area of the lagoon along Wake Island is largely exposed at low tide and represents about $25 \%$ of the total lagoon area. The lagoon has scattered patch reefs on a sandy bottom (Figure 4). Water circulation in the lagoon was severely reduced when the causeway between 


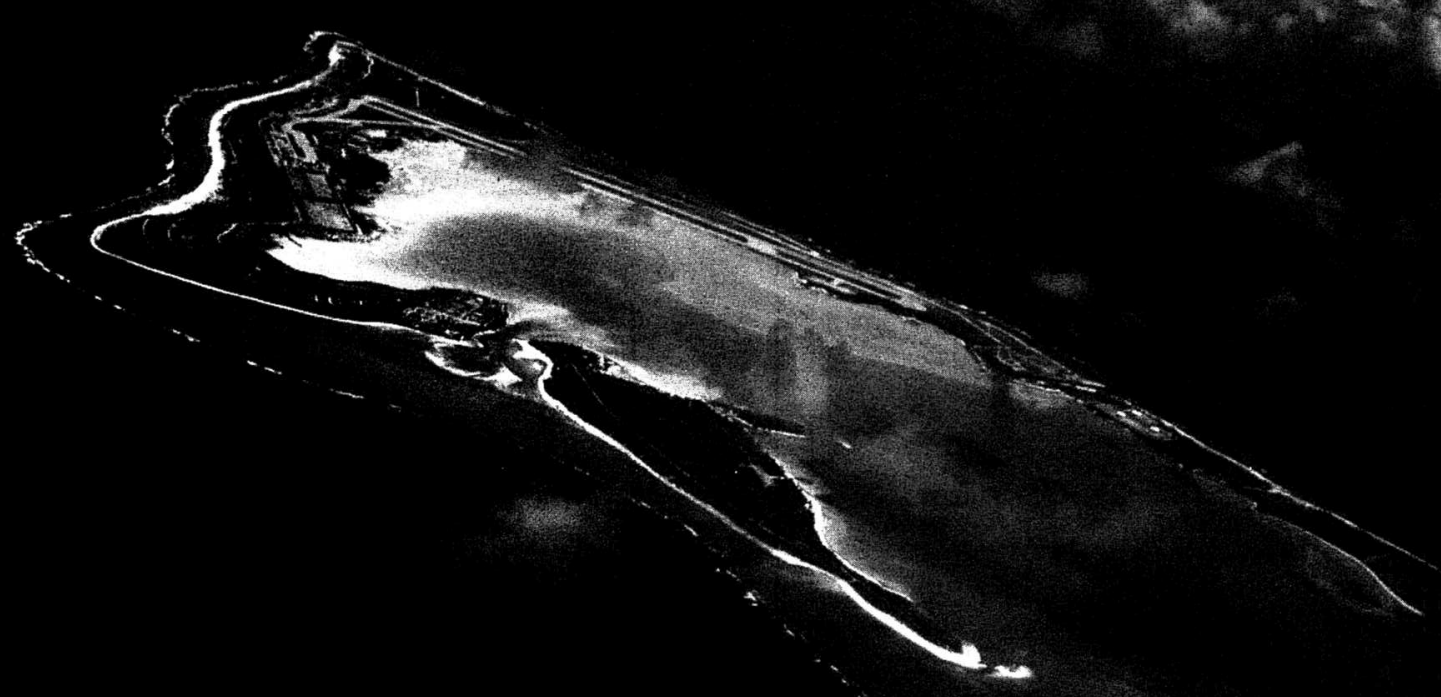

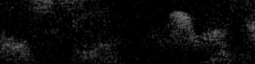

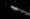

Figure 2. Aerial view of Wake Atoll, looking toward the south. The open reef crest is to the right (west). Peale Island is in the foreground separated from Wake Island by a narrow channel. The northwestern margin of the atoll (foreground) has a wide intertidal reef flat. 


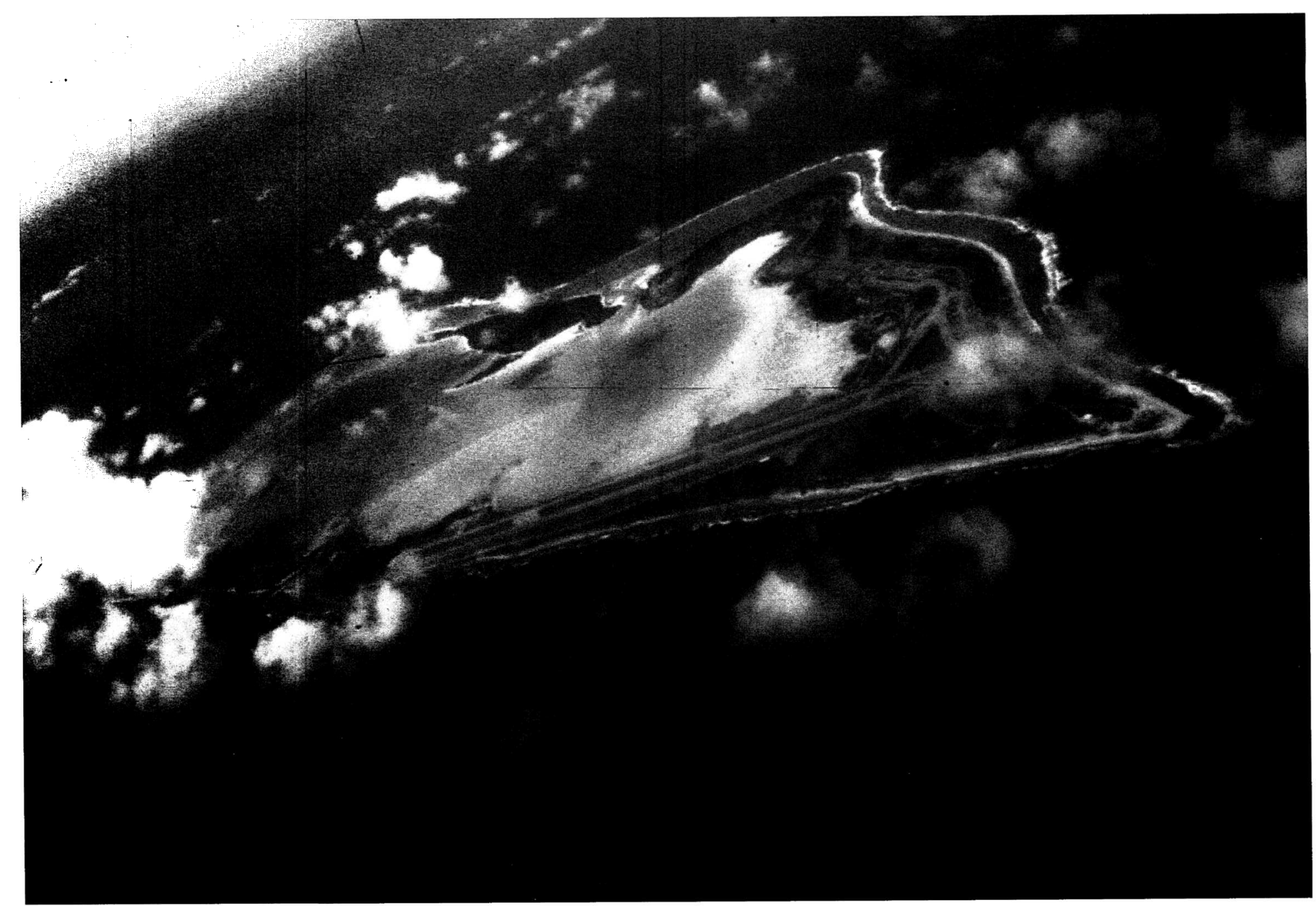

Figure 3. Aerial view of Wake Atoll looking north. The eastern tip of Wake Island is on the right (Peacock Point). This view shows the leeward side of the atoll, which has a very narrow intertidal reef flat that rapidly drops off to deeper depths. 


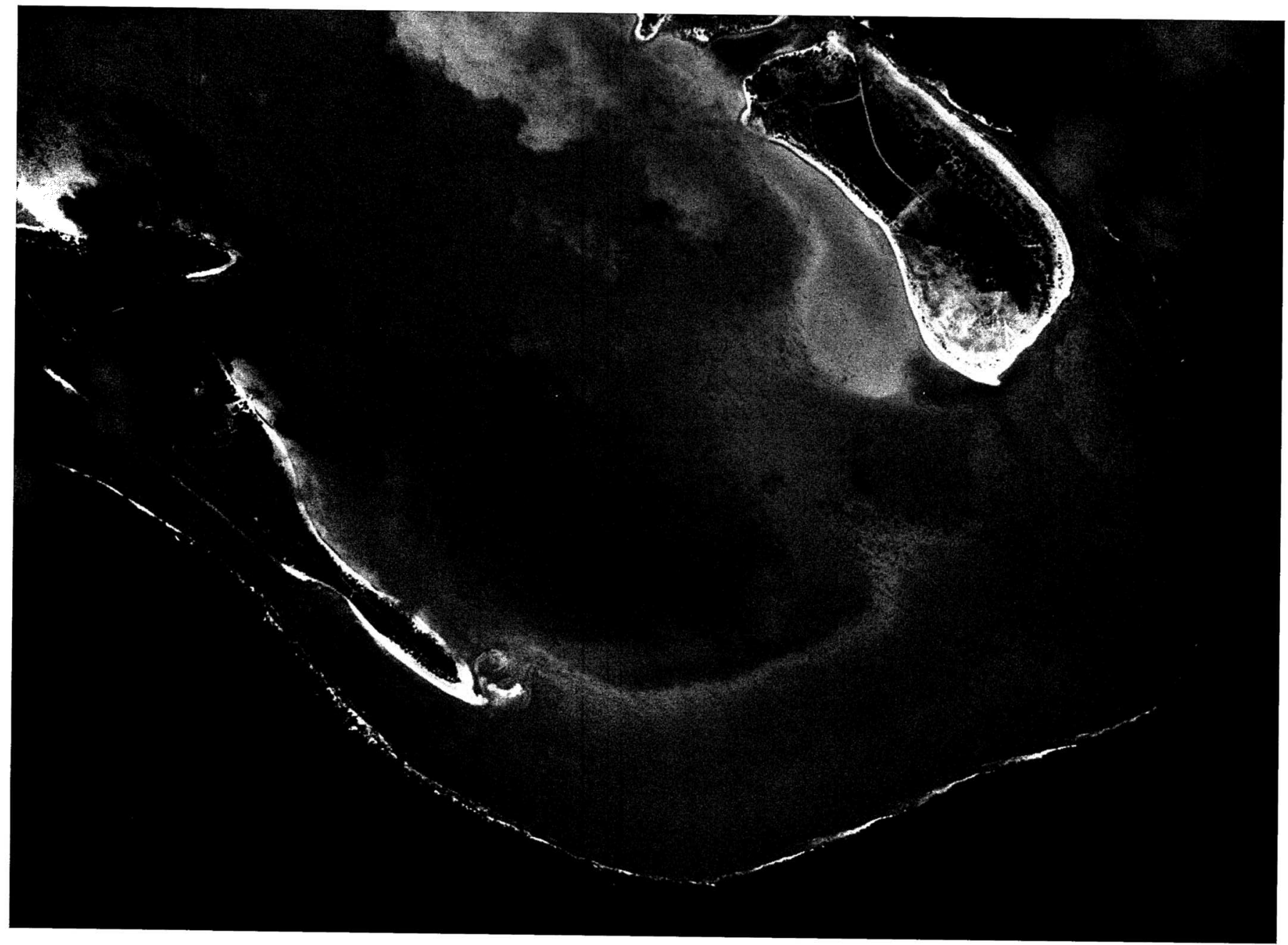
FIGURE 4. Top-down aerial view of the Wake Atoll lagoon showing the western reef, which is only emergent at the lowest tides. The lagoon is mainly
sand flats with few patch reefs. 


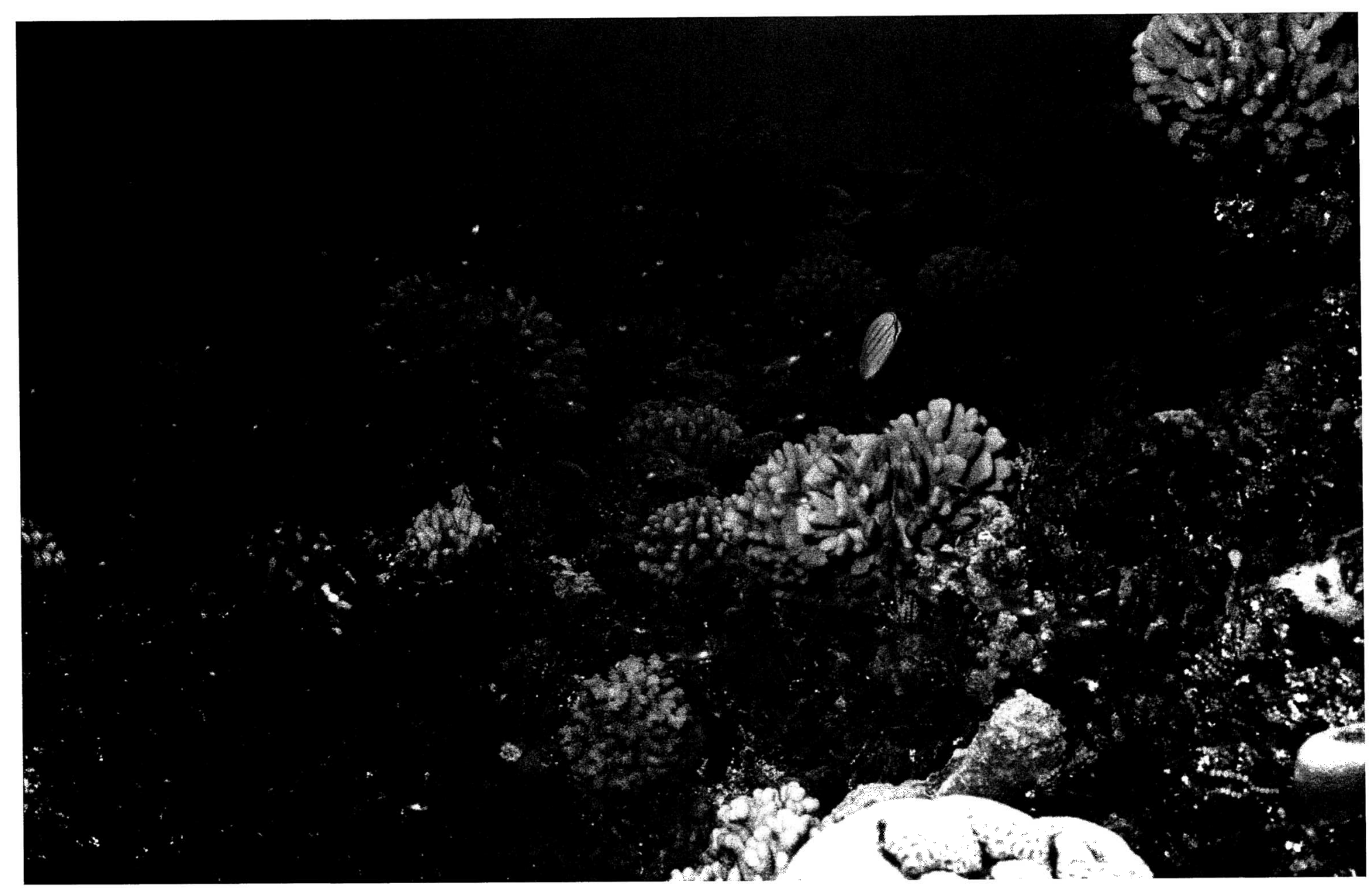

FIGURE 5. Underwater habitat of the outer reef of Wake Atoll showing the dense coral cover. 
Wake and Wilkes Islands was built, resulting in the closure of the small boat harbor inlet into the lagoon. There have been reports of large-scale fish die-offs in the lagoon due to high temperatures when tidal flushing is low resulting in low dissolved oxygen (M. Henz, pers. comm.). The outer reef is densely covered in coral and slopes rapidly to abyssal depths (Figure 5).

The total number of fishes identified from Wake Atoll $(n=321)$ is similar to that found on other isolated central Pacific atolls and islands including Johnston Atoll $(n=301$ [Randall et al. 1985, Kosaki et al. 1991]), Rapa ( $n=268$ [Randall et al. 1990; but many new records recently have been found but not yet reported (J. Randall, pers. comm.)]), and Midway $(n=258$ [Randall et al. 1993]). It has been suggested that the relatively low number of species occurring at these locations could be explained by their small geographic size and limited habitat diversity, combined with their isolation or relatively large distance separating them from other population sources (Randall 1992, 1998b). This comparison is very broad and the islands/atolls vary greatly in their geomorphology. For example, Rapa is a high island, whereas Johnston is an atoll with a large lagoon. Even so the relationship between species diversity and island/atoll isolation and size reveals an interesting and consistent pattern.

The fishes that are found most abundantly at Wake Atoll (Table 2) are typical of those also most abundant on other Pacific Islands (e.g., Kosaki et al. 1991, Randall et al. 1997). The fishes of Wake Atoll can be divided into the following categories: coastal shore fishes (303 species, of which six are elasmobranchs) and offshore pelagic fishes (18 species).

Wake Atoll occupies a unique central location in the northern Pacific and its fauna reflects a mixture of species with different zoogeographic distributions; however, conventional taxonomic classification by species does not always incorporate regional population variation (potential subspecies) that is recognized most readily by color pattern differences (Randall 1998). Thus future research using DNA markers may reveal more regional variation in zoogeography.
TABLE 2

Most Speciose Families of Fishes at Wake Atoll

\begin{tabular}{lccc}
\hline \hline Family & $\begin{array}{c}\text { No. of } \\
\text { Genera }\end{array}$ & $\begin{array}{c}\text { No. of } \\
\text { Species }\end{array}$ & $\begin{array}{c}\text { \% of Total } \\
\text { Fish Fauna }\end{array}$ \\
\hline Labridae & 17 & 38 & 11.8 \\
Gobiidae & 14 & 21 & 6.5 \\
Acanthuridae & 4 & 21 & 6.5 \\
Pomacentridae & 6 & 20 & 6.2 \\
Serranidae & 8 & 19 & 5.9 \\
Chaetodontidae & 4 & 15 & 4.6 \\
Muraenidae & 4 & 15 & 4.6 \\
Holocentridae & 3 & 13 & 4.0 \\
Apogonidae & 3 & 13 & 4.0 \\
Scaridae & 5 & 13 & 4.0 \\
Carangidae & 8 & 12 & 3.7 \\
Blenniidae & 7 & 11 & 3.4 \\
Mullidae & 3 & 8 & 2.5 \\
Balistidae & 5 & 7 & 2.2 \\
Scorpaenidae & 3 & 7 & 2.2 \\
Cirrhitidae & 4 & 6 & 1.9 \\
Tetraodontidae & 2 & 6 & 1.9 \\
\hline
\end{tabular}

It is interesting that a potential ocean current flow pathway seems to exist, at least periodically, between Hawai'i and Wake Atoll. A 90-day oscillation in sea level has been observed intermittently at Wake Atoll based upon two decades of measurements (Mitchum 1995). This oscillation tends to occur about 1.5 yr after an El Niño and appears to be caused by ocean eddies generated off the island of Hawai'i that then move westward and impinge upon Wake Atoll (Mitchum 1995). These ocean eddies are known to contain high concentrations of fish larvae (Lobel and Robinson 1986). We found further evidence of the current flow between Hawai' $i$ and Wake by observing a small sailboat washed ashore with a Hawai'i State registration sticker and from reports of Hawaiian fish aggregation devices (FADS) recovered from Wake by the U.S. Coast Guard (P. Fusco and $M$. Timmons, pers. comm.).

Based upon this evidence, one would expect an overall close faunal affinity between Wake and Hawai'i; however, this does not seem to be the case. For example, a common species, the convict tang, Acanthurus triostegus, is found throughout the Pacific Ocean. The population of $A$. triostegus at Wake Atoll is 

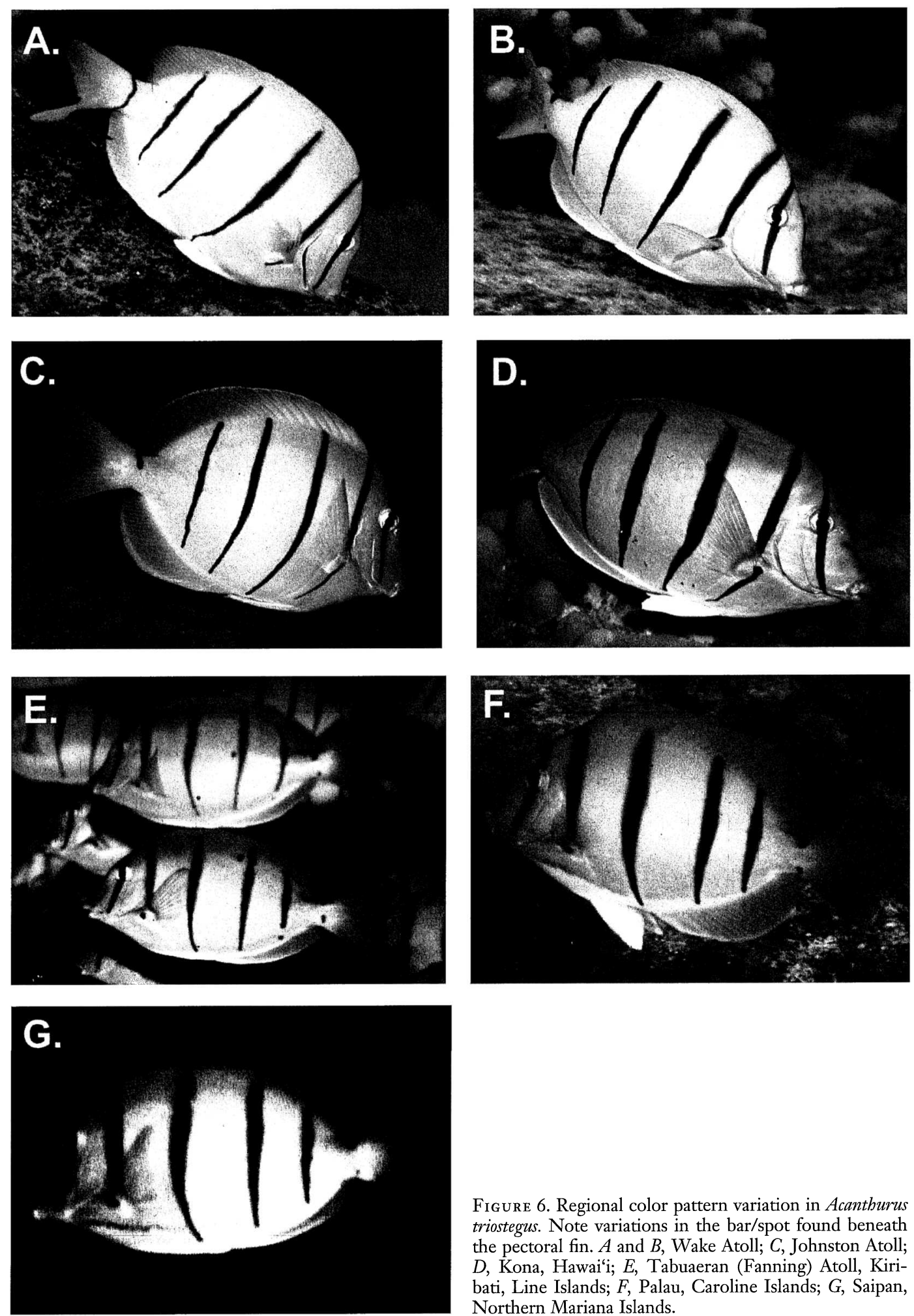

Figure 6. Regional color pattern variation in Acanthurus triostegus. Note variations in the bar/spot found beneath the pectoral fin. $A$ and $B$, Wake Atoll; $C$, Johnston Atoll; $D$, Kona, Hawai'i; $E$, Tabuaeran (Fanning) Atoll, Kiribati, Line Islands; $F$, Palau, Caroline Islands; $G$, Saipan, Northern Mariana Islands. 

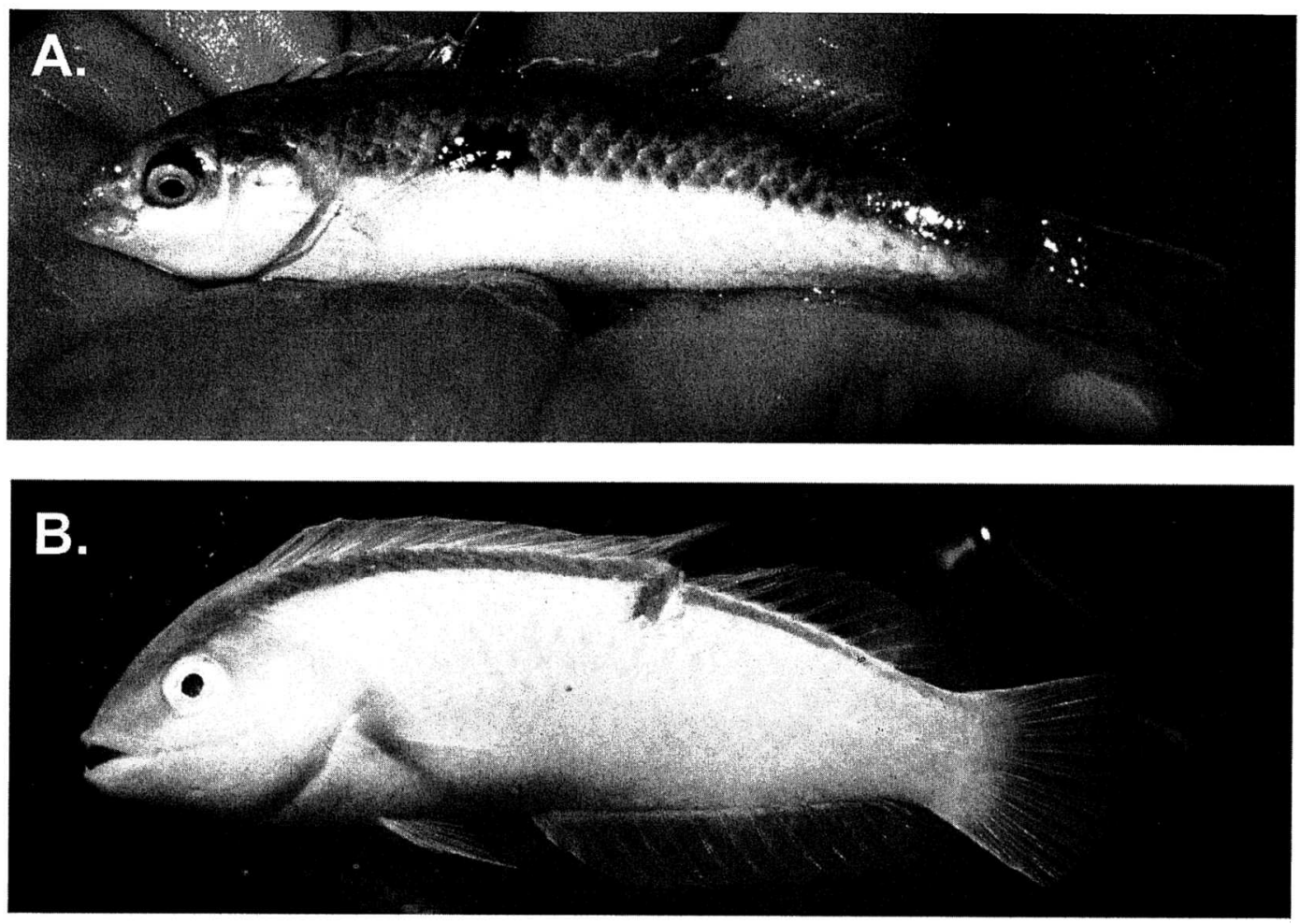

FIGURE 7. Ammolabrus dicrus (Labridae), formerly considered a Hawaiian endemic found only from $\mathrm{O}^{\prime}$ ahu, Hawai'i. It is now found at Wake Atoll. Similar fish have been observed in the Marshall Islands but have not been collected (Randall and Carlson 1997). $A$, Female; $B$, male.

not the same as the distinctive Hawaiian form (which Randall [1956] recognized as a subspecies, A. triostegus sandvicensis). Acanthurus triostegus at Wake Atoll exhibits two color pattern variations: one that is very similar to the populations in the Mariana and Marshall Islands and another that is typical of a variation occurring in Phoenix and Line Island specimens (Figure 6; see also Schultz et al. [1953:625, fig. 90] for illustrations of zoo-

TABLE 3

Zoogeographic Overlap of Wake Atoll Fishes with Those of Nearest Archipelagos

\begin{tabular}{|c|c|c|c|c|c|}
\hline Location & $\begin{array}{c}\% \text { of } \\
\text { Wake Species } \\
\text { in Common }\end{array}$ & Data $^{a}$ & $\begin{array}{c}\% \text { of } \\
\text { Wake Species } \\
\text { at Location }\end{array}$ & $\operatorname{Data}^{b}$ & Reference \\
\hline Hawaiian Islands & 40 & $129 / 321$ & 23 & $129 / 557$ & Randall 1996 \\
\hline Johnston Atoll & 41 & $131 / 321$ & 44 & $131 / 301$ & $\begin{array}{l}\text { Randall et al. } 1985 \text {, } \\
\text { Kosaki et al. } 1991\end{array}$ \\
\hline Northern Mariana Islands & 87 & $279 / 321$ & 30 & $279 / 933$ & Myers 1999 \\
\hline Southern Mariana Islands & 66 & $213 / 321$ & 50 & $213 / 427$ & Myers 1999 \\
\hline Marshall Islands & 82 & $264 / 321$ & 32 & $264 / 817$ & Myers 1999 \\
\hline
\end{tabular}

${ }^{a}$ Number of Wake Atoll species found at location divided by the total number of species at Wake.

${ }^{b}$ Number of Wake Atoll species found at location divided by the total species at location. 

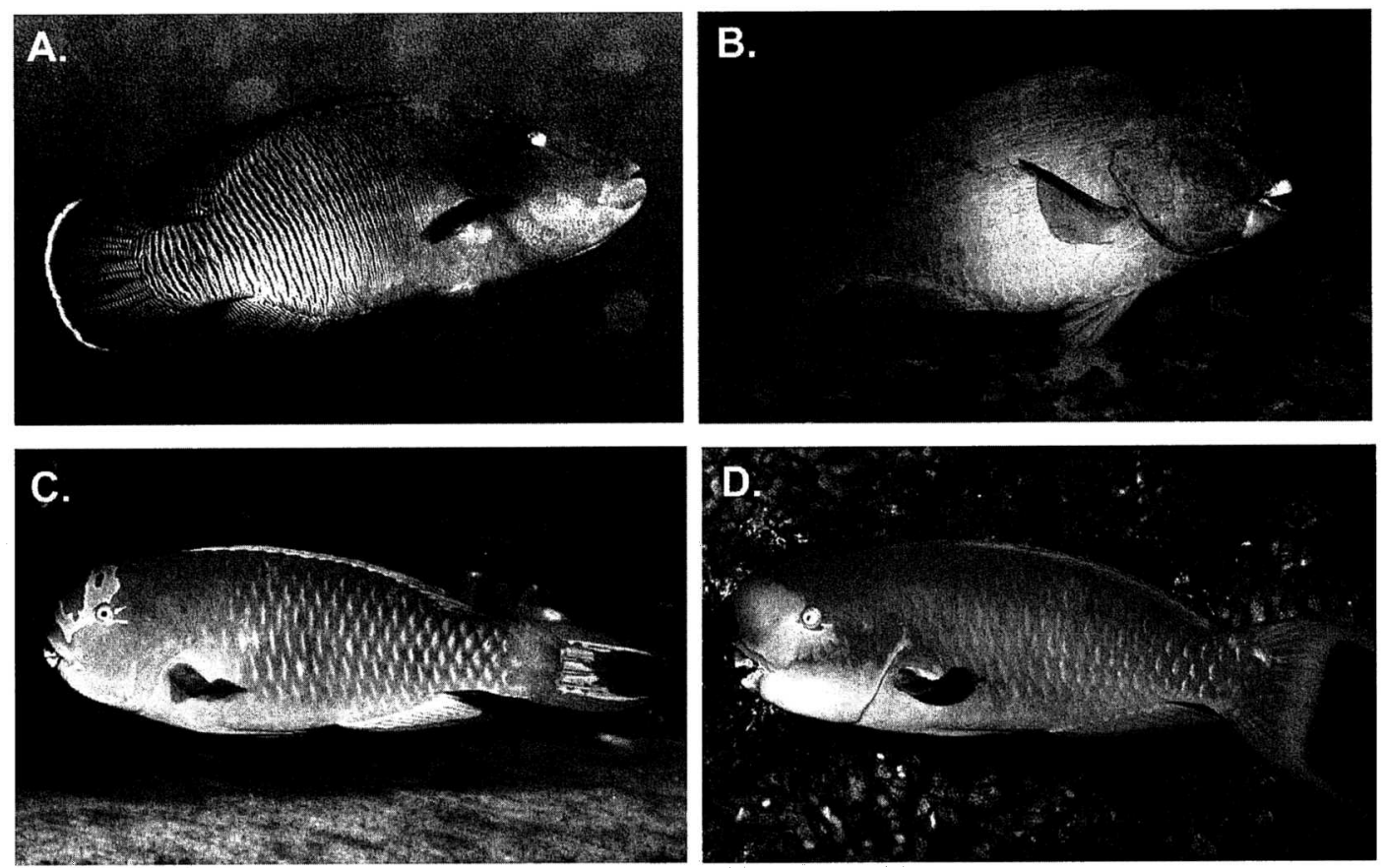

Figure 8. Labroid fishes of special concern for conservation found at Wake Atoll. A, Maori Wrasse, Cheilinus undulatus (Labridae); B, Bumphead Parrotfish, Bolbometopon muricatum (Scaridae); C, Tan-faced Parrotfish, Cblorurus frontalis (Scaridae); D, Pacific Steephead Parrotfish, Chlorurus microrbinos (Scaridae).

geographic color pattern variations in this species).

One species that was previously known only from O'ahu, Hawai'i, is now documented from Wake Atoll: Ammolabrus dicrus
Randall \& Carlson, 1997 (Figure 7). It probably also occurs in the Marshall Islands as well (Randall and Carlson 1997). As noted in the species listing, three other Hawaiian endemics have also been reported from Wake

\section{TABLE 4}

Pacific Fishes of Special Concern for Conservation Found at Wake Atoll

\begin{tabular}{llll}
\hline \hline Family & \multicolumn{1}{c}{ Species } & \multicolumn{1}{c}{ IUCN Category } & \multicolumn{1}{c}{ Common Name } \\
\hline Labridae & Cheilinus undulatus & VUa A1d+2cd & Humphead/Maori Wrasse \\
Scaridae & Bolbometopon muricatum & & Giant Bumphead Parrotfish \\
Scaridae & Chlorurus frontalis & & \\
Scaridae & Chlorurus microrbinus & VU A2d & Giant Grouper \\
Serranidae & Epinephalus lanceolatus & Shortlist for assessment & Camouflage Grouper \\
Serranidae & Epinephalus polyphekadion & Shortlist for assessment & Yellow-edged Lyretail \\
Serranidae & Variola louti & S &
\end{tabular}

${ }^{a}$ Vulnerable based on the following IUCN (2001) criteria: A1d, reduction in population size based on actual or potential levels of exploitation; 2cd, an observed, estimated, inferred, or suspected population size reduction of $30 \%$ over the last 10 yr or three generations, whichever is the longer, where the reduction or its causes may not have ceased OR may not be understood OR may not be reversible, based on a decline in area of occupancy, extent of occurrence, and/or quality of habitat and actual or potential levels of exploitation; A2d, an observed, estimated, inferred, or suspected population size reduction of $30 \%$ over the last $10 \mathrm{yr}$ or three generations, whichever is the longer, where the reduction or its causes may not have ceased OR may not be understood OR may not be reversible, based on actual or potential levels of exploitation. 


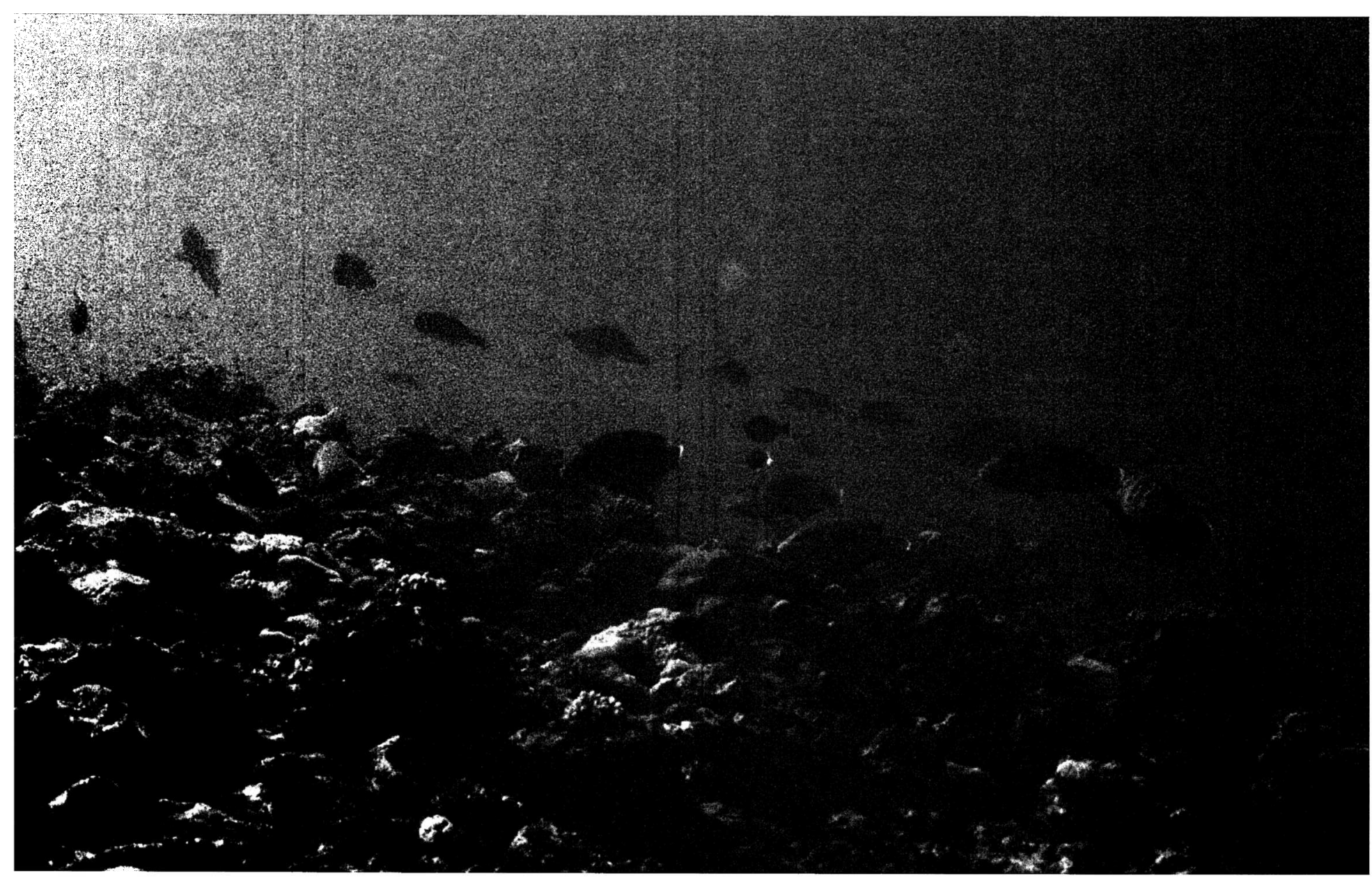

Figure 9. This underwater scene shows a school of large parrotfish in the background and at least six large Maori Wrasses (Cbeilinus undulatus). Such scenes are no longer observed on other Pacific atolls subjected to commercial fishing. 
Atoll, but these are probably misidentifications (Eviota epiphanes, Entomacrodus marmoratus, Sebastipistes ballieui).

The Wake Atoll fish fauna has the most species in common with that of the Mariana Islands. A zoogeographic comparison of the Wake Atoll fish fauna with those of the four nearest neighboring archipelagos was made using our checklist and the species distribution data compiled from the literature. This zoogeographic analysis indicates that the greatest species overlap occurs with the northern Mariana Islands (87\%) and the Marshall Islands (82\%). Although the southern Mariana Islands have a low percentage of species in common with Wake $(66 \%)$, the southern Mariana Islands had a much greater proportion of their total fish fauna also found at Wake than did the other five locations examined (Table 3). This pattern is corroborated by the Acanthurus triostegus example.

Another unique aspect of Wake Atoll is the protection of its natural resources, in particular fisheries, by the U.S. Department of Defense. This is achieved primarily through the exclusion of commercial fishing ventures from Wake and the ban on reef fish spearfishing. Our surveys discovered four labroid fishes (Figure 8) and three groupers on Wake's reefs that are threatened by exploitation elsewhere in the Pacific (Table 4). In particular, the Maori Wrasse (Cheilinus undulatus), which is listed on the IUCN Red List (2001) (IUCN Grouper and Wrasse Specialist Group [http://www.hku.hk/ecology/ GroupersWrasses/iucnsg/doc/species.html]) as vulnerable to overexploitation, is abundant at Wake (Figure 9). The Maori Wrasse is a prized food fish and is targeted for the live reef fish trade (LRFT) in Hong Kong (Donaldson and Sadovy 2001). These fishes of concern are not fished from Wake waters and therefore population numbers may be considered pristine; however, fishing of other species does occur by the residents and visitors of Wake. For example, very few sharks were observed, which is unusual for a remote Pacific atoll. The local workers reportedly fish occasionally for sharks as well as for other reef species, but whether this has negatively impacted the population was not determined. This emphasizes the need for continued protection of Wake Atoll's coral reef ecosystem.

\section{ACKNOWLEDGMENTS}

We are especially grateful for the enormous level of assistance provided by John Randall, Helen Randall, and Arnold Suzumoto of the врвм. This paper could not have been completed without their help. We also thank John McCosker, William Eschmeyer, and Jon Fong of the Department of Ichthyology, cas; Karsten Hartell of the MCZ; the late Eugenie Böhlke and Mark Sabaj of the Department of Ichthyology, ANsP; and Rob Myers for their assistance. We are grateful for the logistic support on Wake Island provided by Paul Fusco, Mike Timmons, and Mark Henz. Logistic support was also provided by Mark Ingoglia and Jeff Klein of the U.S. Air Force, Pacific. We thank Mike Cermac for help with the fish distribution database.

\section{Literature Cited}

Allen, G. R. 1991. Damselfishes of the world. Aquarium Systems, Mentor, Ohio.

Allen, G. R., and J. E. Randall. 1977. Review of the sharpnose pufferfishes (subfamily Canthigasterinae) of the Indo-Pacific. Rec. Aust. Mus. 30 (17): 475-517.

Böhlke, E. B., and J. E. Randall. 2000. A review of the moray eels (Anguilliformes: Muraenidae) of the Hawaiian Islands, with descriptions of two new species. Proc. Acad. Nat. Sci. Phila. 150:203-278.

Collette, B. B. 1974. Geographic variation in the central Pacific halfbeak, Hyporbamphus acutus (Gunther). Pac. Sci. 28:111-122.

Donaldson, T. J., and Y. Sadovy. 2001. Threatened fishes of the world: Cheilinus undulatus Ruppell 1835 (Labridae). Environ. Biol. Fishes 62:428.

Eschmeyer, W. N., and J. E. Randall. 1975. The scorpaenid fishes of the Hawaiian Islands including new species and new records (Pisces: Scorpaenidae). Proc. Calif. Acad. Sci. 40 (11): 265-334.

Fowler, H. W. 1928. The fishes of Oceania. Mem. Bernice P. Bishop Mus. 10. 
Fowler, H. W., and S. C. Ball. 1925. Fishes of Hawaii, Johnston Island, and Wake Island. Bernice P. Bishop Mus. Bull. 26:131.

Greenfield, D. W. 2001. Revision of the Apogon erytbrinus complex (Teleostei: Apogonidae). Copeia 2001 (2): 459-472.

IUCN. 2001. IUCN Red List categories and criteria: Version 3.1. IUCN Species Survival Commission. IUCN, Gland, Switzerland, and Cambridge, UK.

Karnella, S. J., and E. A. Lachner. 1981. Three new species of the Eviota epiphanes group having vertical trunk bars (Pisces: Gobiidae). Proc. Biol. Soc. Wash. 94 (1): 264-275.

Kerr, L. M., and P. S. Lobel, eds. 2000. Department of Defense coral reef protection implementation plan. U.S. Coral Reef Task Force Report. Government Printing Office, Washington, D.C.

Kosaki, R. K., R. L. Pyle, J. E. Randall, and D. K. Irons. 1991. New records of fishes from Johnston Atoll, with notes on biogeography. Pac. Sci. 45:186-203.

Lachner, E. A., and S. J. Karnella. 1980. Fishes of the Indo-Pacific genus Eviota with descriptions of eight new species (Teleostei: Gobiidae). Smithson. Contrib. Zool. 315.

Lobel, P. S., and A. R. Robinson. 1986. Transport and entrapment of fish larvae by ocean mesoscale eddies and currents in Hawaiian waters. Deep-Sea Res. 33 (4): 483-500.

McCosker, J. E., and R. H. Rosenblatt. 1993. A revision of the snake eel genus Myrichthys (Anguilliformes: Ophichthidae) with the description of a new eastern Pacific species. Proc. Calif. Acad. Sci. 48 (8): 153169.

McCosker, J. E., K. Hatooka, K. Sasaki, and J. T. Moyer. 1984. Japanese morays of the genus Uropterygius. Jpn. J. Ichthol. 31 (3): 261-267.

Mitchum, G. T. 1995. The source of 90-day oscillations at Wake Island. J. Geophys. Res. 100 (2): 2459-2475.

Myers, R. F. 1999. Micronesian reef fishes. 3rd ed. Coral Graphics, Guam.

Randall, J. E. 1956. A revision of the surgeon fish genus Acantburus. Pac. Sci. 10:159235.

1992. Endemism of fishes in Oceania. UNEP: Coastal resources and systems of the Pacific Basin: Investigation and steps toward protective management. UNEP Regional Seas Reports and Studies no. 147:55-67.

1996. Shore fishes of Hawaii. Natural World Press, Vida, Oregon.

1998a. Revision of the Indo-Pacific squirrelfishes (Beryciformes: Holocentridae: Holocentrinae) of the genus Sargocentron, with descriptions of four new species. Indo-Pac. Fishes 27.

.1998b. Zoogeography of shore fishes of the Indo-Pacific region. Zool. Stud. 37 (4): 227-268.

1999. Revision of the Indo-Pacific labrid fishes of the genus Coris, with descriptions of five new species. Indo-Pac. Fishes 29.

Randall, J. E., and B. A. Carlson. 1997. Ammolabrus dicrus, a new genus and species of labrid fish from the Hawaiian Islands. Pac. Sci. 51:29-35.

Randall, J. E., and K. D. Clements. 2001. Second revision of the surgeonfish genus Ctenochaetus (Peciformes: Acanthuridae), with descriptions of two new species. Indo-Pac. Fishes 32.

Randall, J. E., and D. W. Greenfield. 1996. Revision of the Indo-Pacific holocentrid fishes of the genus Myripristis, with descriptions of three new species. Indo-Pac. Fishes 25.

Randall, J. E., and P. C. Heemstra. 1991. Revision of Indo-Pacific groupers (Perciformes: Serranidae: Epinephelinae), with descriptions of five new species. Indo-Pac. Fishes 20.

Randall, J. E., and P. S. Lobel. Xyrichthys balsteadi, a new labrid fish from the central and western Pacific. Bull.Mar. Sci. (in press).

Randall, J. E., and R. F. Myers. 2002. Parupeneus insularis, a new central Pacific species of goatfish (Perciformes: Mullidae) of the $P$. trifasciatus complex. Zool. Stud. 41 (4): 431-440.

Randall, J. E., and H. A. Randall. 2001. Review of the fishes of the genus Kublia 
(Perciformes: Kuhliidae) of the central Pacific. Pac. Sci. 55:227-256.

Randall, J. E., P. S. Lobel, and E. H. Chave. 1985. Annotated checklist of the fishes of Johnston Island. Pac. Sci. 39:24-80.

Randall, J. E., M. L. Bauchot, and M. Desoutter. 1987. Heliases ternatensis Bleeker, 1856 (currently Chromis ternatensis; Osteichthys, Perciformes): Proposed conservation, and adoption of the name Chromis viridis (Cuvier, 1830) for the fish commonly called $C$. caerulea (Cuvier, 1830). Case 2516. Bull. Zool. Nomencl. 44 (4): 248-250.

Randall, J. E., C. L. Smith, and M. N. Feinberg. 1990. Report on fish collections from Rapa, French Polynesia. Am. Mus. Novit. 2966.

Randall, J. E., J. L. Earle, R. L. Pyle, J. D. Parrish, and T. Hayes. 1993. Annotated checklist of the fishes of Midway Atoll, Northwestern Hawaiian Islands. Pac. Sci. 47:356-400.

Randall, J. E., H. Ida, K. Kato, R. L. Pyle, and J. L. Earle. 1997. Annotated checklist of the inshore fishes of the Ogasawara Islands. Natl. Sci. Mus. Tokyo Monogr. 11.

Schultz, L. P., E. S. Herald, E. A. Lachner, A. D. Welander, and L. P. Woods. 1953. Fishes of the Marshall and Marianas Islands. U.S. Natl. Mus. Bull. 202 (1).

Springer, V. G., and J. T. Williams. 1990. Widely distributed Pacific Plate endemics and lowered sea-level. Bull. Mar. Sci. 47 (3): 631-640.

. 1994. The Indo-West Pacific blenniid fish genus Istiblennius reappraised: A revision of Istiblennius, Blenniella, and $\mathrm{Pa}$ ralticus, new genus. Smithson. Contrib. Zool. 565.

Williams, J. T. 1988. Revision and phylogenetic relationships of the blenniid fish genus Cirripectes. Indo-Pac. Fishes. 17.

Yoshino, T., T. Kon, and S. Okabe. 1999. Review of the genus Limnichtbys (Perciformes: Creedidae) from Japan, with description of a new species. Ichthyol. Res. 46 (1): 73-83. 